\title{
Discrete group actions on Stein domains in complex Lie groups
}

\author{
Dehbia Achab, Frank Betten ${ }^{\dagger}$, Bernhard Krötz*
}

\begin{abstract}
This paper deals with the analytic continuation of holomorphic automorphic forms on a (Hermitian) Lie group $G$. We prove that for any discrete subgroup $\Gamma$ of $G$ there always exists a non-trivial holomorphic automorphic form, i.e., there exists a $\Gamma$-spherical unitary highest weight representation of $G$. Holomorphic automorphic forms have the property that they analytically extend to holomorphic functions on a complex Ol'shanskiǔ semigroup $S \subseteq G_{\mathbb{C}}$. As an application we prove that the bounded holomorphic functions on $\Gamma \backslash S \subseteq \Gamma \backslash G_{\mathbb{C}}$ separate the points.
\end{abstract}

\section{Introduction}

Let $G$ be a connected Lie group sitting in its complexification $G_{\mathbb{C}}$. Let $\Gamma<G$ be any discrete subgroup. Then $\Gamma$ acts freely and properly discontinuously on $G_{\mathbb{C}}$ and the quotient $\Gamma \backslash G_{\mathbb{C}}$ is a complex manifold. It is reasonable to ask which such homogeneous complex manifolds are Stein. If $G$ is nilpotent, then $\Gamma \backslash G_{\mathbb{C}}$ is Stein by a theorem of Gilligan and Huckleberry (cf. [GiHu78]). We call a Lie algebra $\mathfrak{g}$ weakly elliptic, if all operators $\operatorname{ad}(X), X \in \mathfrak{g}$, have imaginary spectrum and note that all nilpotent and many solvable Lie algebras are weakly elliptic. Then Loeb has shown in [Lo84] that $\Gamma \backslash G_{\mathbb{C}}$ is Stein whenever the Lie algebra $\mathfrak{g}$ of $G$ is weakly elliptic. But if $G$ is semisimple and $\Gamma$ is a lattice, then a theorem of Barth and Otte (cf. [BaOt73]) implies that the only holomorphic functions on $\Gamma \backslash G_{\mathbb{C}}$ are the constants; in particular $\Gamma \backslash G_{\mathbb{C}}$ is not Stein. Therefore in general the $G \times G$-biinvariant complex manifold $G_{\mathbb{C}}$ is too large for $\Gamma \backslash G_{\mathbb{C}}$ carrying a rich complex structure. In this paper we will identify an open $G \times G$-invariant Stein domain $S \subseteq G_{\mathbb{C}}$ for which we prove that there is a rich supply of automorphic forms on $\Gamma \backslash G$ which holomorphically extend to $\Gamma \backslash S$. In an important example we will prove that $\Gamma \backslash S$ is Stein.

Let us illustrate the situation for the example $G=\mathrm{SU}(p, q)$. Then $G$ can be defined as the invariance group of a hermitian form $\langle\cdot, \cdot\rangle_{p, q}$ on $\mathbb{C}^{n}, n=p+q$, and we have $G_{\mathbb{C}}=\operatorname{Sl}(n, \mathbb{C})$. The contraction semigroup of the form $\langle\cdot, \cdot\rangle_{p, q}$

$$
S:=\left\{g \in G_{\mathbb{C}}:\langle g \cdot v, g \cdot v\rangle_{p, q}<\langle v, v\rangle_{p, q} \text { for all } v \in \mathbb{C}^{n}, v \neq 0\right\}
$$

is easily seen to be an open $G \times G$-invariant domain in $G_{\mathbb{C}}$, a so-called complex Ol'shanskiu semigroup. In general one can define complex Ol'shanskiǔ semigroups for every connected Lie

${ }^{\dagger}$ Supported by the DFG-grant BE 2080/1-1, * Supported by the DFG-project HI 412/5-2 
group, whenever its Lie algebra admits an open convex weakly elliptic $\operatorname{Ad}(G)$-invariant cone $\varnothing \neq W \subseteq \mathfrak{g}$. If $\mathfrak{g}$ is simple, this means that $\mathfrak{g}$ is either hermitian or compact, but we also have many non-reductive examples as the Jacobi-algebra $\mathfrak{h}_{n} \rtimes \mathfrak{s p}(n, \mathbb{R})$ with $\mathfrak{h}_{n}$ the $2 n+1$-dimensional Heisenberg algebra (cf. [Ne99a] and Example 1.3 below). The complex Ol'shanskiù semigroup associated to $G$ and $W$ is given by $S=G \operatorname{Exp}(i W)$.

In [Ne98, Ne99b] Neeb settled a conjecture of Gindikin by showing that all complex Ol'shanskiı̌ semigroups and their symmetric space analogues are Stein manifolds. Let now $\Gamma<G$ be any discrete subgroup. In this paper we investigate the quotients $\Gamma \backslash S$. These manifolds were first considered by the first author for $G=\operatorname{Sl}(2, \mathbb{R})$ in the context of Hardy spaces on $\Gamma \backslash S$ (cf. [Ach99]).

Let us in the following assume that $\operatorname{Ad}(G)$ is closed in $\operatorname{Aut}(\mathfrak{g})$ and that $W$ is pointed, which both are very natural assumptions in our context. Then one of our main results is:

Theorem A. (cf. 4.7) If $\Gamma<G$ is a discrete subgroup of $G$, then:

(i) $\operatorname{Hol}(\Gamma \backslash S)$ separates the points of $\Gamma \backslash S$.

(ii) If $\Gamma<G$ is cocompact, then the bounded holomorphic functions on $\Gamma \backslash S$ separate the points. In particular, the Caratheodory semimetric on $\Gamma \backslash S$ is a metric.

We construct holomorphic functions on $\Gamma \backslash S$ with techniques from representation theory.

Let $\mathfrak{t} \subseteq \mathfrak{g}$ denote a compactly embedded Cartan subalgebra of $\mathfrak{g}$. If $\left(\pi_{\lambda}, \mathcal{H}_{\lambda}\right)$ is a unitary highest weight representation of $G$ with highest weight $\lambda \in i t^{*}$, then one has a holomorphic extension to a representation of the complex Ol'shanskiu semigroup $S$ which has $G$ as some sort of Shilov boundary (cf. [Ne99a, Ch. XIV]). We obtain holomorphic functions on $\Gamma \backslash S$ by taking matrix-coefficients with $\Gamma$-fixed hyperfunction vectors; i.e., we take

$$
\theta_{v, \eta}: \Gamma \backslash S \rightarrow \mathbb{C}, \quad \Gamma s \mapsto\left\langle\pi_{\lambda}(s) . v, \eta\right\rangle,
$$

with $v \in \mathcal{H}_{\lambda}$ and $\eta \in \mathcal{H}_{\lambda}^{-\omega}$ a $\Gamma$-fixed element. Here $\mathcal{H}_{\lambda}^{-\omega}$ denotes the Fréchet $G$-module of hyperfunction vectors of $\left(\pi_{\lambda}, \mathcal{H}_{\lambda}\right)$, the strong antidual of the analytic vectors $\mathcal{H}_{\lambda}^{\omega}$. Note that for $v$ being a highest weight vector the function $\theta_{v, \eta}$ is an analytic continuation of the (anti)holomorphic automorphic form $\left.\theta_{v, \eta}\right|_{G}$ (cf. [Bo66]).

Our considerations from above show that one gets many holomorphic functions on $\Gamma \backslash S$ provided $\Gamma$-spherical unitary highest weight representations $\left(\pi_{\lambda}, \mathcal{H}_{\lambda}\right)$ of $G$ do exist. Our central result is as follows:

Theorem B. (cf. 3.8 - 3.11) Let $\Gamma$ be an arbitrary discrete subgroup of $G$ and assume that $G$ has compact center. Denote by $\Lambda \subseteq i t^{*}$ the spectrum of the $L^{1}$-Bergman space $\mathcal{B}^{1}(S)$ on $S$. Then the following assertions hold:

(i) For all $\lambda \in \Lambda$ and $v \in \mathcal{H}_{\lambda}^{\omega}$ the Poincaré series of $v$

$$
P(v):=\sum_{\gamma \in \Gamma} \pi_{\lambda}(\gamma) \cdot v
$$

converges in the module of hyperfunction vectors $\mathcal{H}_{\lambda}^{-\omega}$ to a $\Gamma$-fixed element.

(ii) For all but finitely many $\lambda \in \Lambda$ we have $P\left(v_{\lambda}\right) \neq 0$, where $v_{\lambda}$ is a highest weight vector of $\left(\pi_{\lambda}, \mathcal{H}_{\lambda}\right)$.

Part (i) is obtained from integral geometric observations in the Bergman space $\mathcal{B}^{1}(S)$ together with the Plancherel theorem for $\mathcal{B}^{2}(S)$ (cf. [Kr98a]).

To get a feeling for the contents of (ii) we explain it for the example $G=\mathrm{SU}(1,1)$. Write $D \cong G / K$ for the open unit disc and write elements of $\Gamma$ as $\gamma=\left(\begin{array}{ll}a_{\gamma} & b_{\gamma} \\ c_{\gamma} & d_{\gamma}\end{array}\right)$. Then $\mathcal{H}_{\lambda}^{-\omega}$ can 
be naturally be identified with $\operatorname{Hol}(D)$ and we have

$$
P\left(v_{\lambda}\right)(z):=\sum_{\gamma \in \Gamma}\left(c_{\gamma} z+d_{\gamma}\right)^{\lambda} \quad(z \in D) .
$$

Here the parameter $-\lambda$ ranges in the integers with $\lambda \geq 3$. The idea for the proof lies in an analytic continuation of $P\left(v_{\lambda}\right)$ in the parameter $\lambda$ together with a clever argument that this analytic continuation is non-zero.

Statements related to (ii) were also proved by Siegel (cf. [Fr83]). He considers scalar valued Poincaré series (this means that the minimal $K$-type of $\left(\pi_{\lambda}, \mathcal{H}_{\lambda}\right)$ is one-dimensional) and proves that for a given $\Gamma$ there always exists a $K$-finite vector $v$ such that $P(v) \neq 0$. Siegel's proof relies heavily on the fact that $D$ is a bounded domain while in our approach this is immaterial (for the Jacobi group $D$ is unbounded). Further our argument does not need that the Poincaré series is scalar valued. Finally, Siegel's method does not tell for which $K$-finite vector $v$ one has $P(v) \neq 0$ while our techniques yield $P\left(v_{\lambda}\right) \neq 0$ for the distinguished highest weight vectors $v_{\lambda}$. We would also like to mention that (ii) partly solves an open problem posed by Wells and Wolf (cf. [WeWo79]; see also [WaWo83]).

In Chapter 4 we show that the analytically continued automorphic functions $\theta_{v, \eta}$ vanish at infinity:

Theorem C. (cf. 4.6) If $\Gamma<G$ is cocompact and $v \in \mathcal{H}_{\lambda}^{\omega}$, then we have

$$
\lim _{\substack{s \rightarrow \infty \\ s \in \Gamma \backslash \bar{S}}} \theta_{v, \eta}(s)=0 .
$$

It is our impression that this result in combination with Theorem A might yield the Stein property of $\Gamma \backslash S$ in the cocompact case (cf. Conjecture 5.3 below for a more detailed discussion).

For $G=\operatorname{Sl}(2, \mathbb{R})$ the existence of a non-vanishing holomorphic cusp form (the discriminant) allows to prove the following:

Theorem D. (cf. 5.1 - 5.2) For $G=\mathrm{Sl}(2, \mathbb{R})$ and all subgroups $\Gamma<\mathrm{Sl}(2, \mathbb{Z})$ the quotients $\Gamma \backslash S$ are Stein.

The authors would like to thank Karl-Hermann Neeb for his very useful remarks.

\section{Complex Ol'shanskiǔ semigroups and their representations}

In this first section we introduce complex Ol'shanskiǔ semigroups, which may be thought of as complex Lie subsemigroups of complex Lie groups. Then we recall the basic facts concerning their holomorphic representations and the characterization of the irreducible ones by highest weight representations. The concepts will be illustrated with some importnat examples (cf. Example 1.3 and Remark 1.7).

\section{Complex Ol'shanskiı̌ semigroups}

Throughout $\mathfrak{g}$ denotes a finite dimensional real Lie algebra. The concept of a complex Ol'shanskil semigroup is closely related to the concept of elliptic elements in a real Lie algebra $\mathfrak{g}$. Recall that an element $X \in \mathfrak{g}$ is called elliptic if ad $X$ is semsimple with purely imaginary spectrum. Accordingly we call a subset $W \subseteq \mathfrak{g}$ elliptic if all its elements are elliptic. 
Definition 1.1. (Complex Ol'shanskiĭ semigroups, cf. [Ne99a, Ch. XI]) Let $\varnothing \neq W \subseteq \mathfrak{g}$ be an open convex elliptic cone and $\bar{W}$ its closure. Let $\widetilde{G}$, respectively $\widetilde{G}_{\mathbb{C}}$, be the simply connected Lie groups associated to $\mathfrak{g}$, respectively $\mathfrak{g}_{\mathbb{C}}$, and set $G_{1}:=\langle\exp \mathfrak{g}\rangle \subseteq \widetilde{G}_{\mathbb{C}}$. Then Lawson's Theorem says that the subset $\Gamma_{G_{1}}(\bar{W}):=G_{1} \exp (i \bar{W})$ is a closed subsemigroup of $\widetilde{G}_{\mathbb{C}}$ and the polar map

$$
G_{1} \times \bar{W} \rightarrow \Gamma_{G_{1}}(\bar{W}), \quad(g, X) \mapsto g \exp (i X),
$$

is a homeomorphism.

Now the universal covering semigroup $\Gamma_{\widetilde{G}}(\bar{W}):=\widetilde{\Gamma}_{G_{1}}(\bar{W})$ has a similar structure. We can lift the exponential function exp: $\mathfrak{g}+i \bar{W} \rightarrow \Gamma_{G_{1}}(\bar{W})$ to an exponential mapping Exp: $\mathfrak{g}+i \bar{W} \rightarrow$ $\Gamma_{\widetilde{G}}(\bar{W})$ with $\operatorname{Exp}(0)=\mathbf{1}$ and thus obtain a polar map $\widetilde{G} \times \bar{W} \rightarrow \Gamma_{\widetilde{G}}(\bar{W}),(g, X) \mapsto g \operatorname{Exp}(i X)$ which is a homeomorphism.

If $G$ is any connected Lie group associated to $\mathfrak{g}$, then $\pi_{1}(G)$ is a discrete central subgroup of $\Gamma_{\widetilde{G}}(\bar{W})$ and we obtain a covering homomorphism $\Gamma_{\widetilde{G}}(\bar{W}) \rightarrow \Gamma_{G}(\bar{W}):=\Gamma_{\widetilde{G}}(\bar{W}) / \pi_{1}(G)$. It is easy to see that there is also a polar map $G \times \bar{W} \rightarrow \Gamma_{G}(\bar{W}),(g, X) \mapsto g \operatorname{Exp}(i X)$, which is a homeomorphism. The semigroups of the type $\Gamma_{G}(\bar{W})$ are called complex Ol'shanskiu semigroups.

The subset $\Gamma_{G}(W) \subseteq \Gamma_{G}(\bar{W})$ is an open semigroup carrying a complex manifold structure such that the multiplication is holomorphic. Moreover there is an involution on $\Gamma_{G}(\bar{W})$ given by

$$
{ }^{*}: \Gamma_{G}(\bar{W}) \rightarrow \Gamma_{G}(\bar{W}), \quad s=g \operatorname{Exp}(i X) \mapsto s^{*}=\operatorname{Exp}(i X) g^{-1},
$$

being antiholomorphic on $\Gamma_{G}(W)$. Thus both $\Gamma_{G}(W)$ and $\Gamma_{G}(\bar{W})$ are involutive semigroups.

From now on we denote by $S$ an open complex Ol'shanskiu semigroup $\Gamma_{G}(W)$ and by $\bar{S}$ its "closure" $\Gamma_{G}(\bar{W})$.

If $\mathfrak{l}$ is a subalgebra of a Lie algebra $\mathfrak{g}$, then we write $\operatorname{Inn}_{\mathfrak{g}}(\mathfrak{l}) \subseteq \operatorname{Aut}(\mathfrak{g})$ for the subalgebra of the automorphism group $\operatorname{Aut}(\mathfrak{g})$ of $\mathfrak{g}$ which is generated by the elements $e^{\operatorname{ad} X}, X \in \mathfrak{l}$. If $\mathfrak{l}=\mathfrak{g}$, then we also write $\operatorname{Inn}(\mathfrak{g})$ instead of $\operatorname{Inn}_{\mathfrak{g}}(\mathfrak{g})$.

A subalgebra $\mathfrak{a} \subseteq \mathfrak{g}$ is said to be compactly embedded, if $\operatorname{Inn}_{\mathfrak{g}}(\mathfrak{a})$ is relatively compact in $\operatorname{Aut}(\mathfrak{g})$. Note that a subalgebra is compactly embedded if and only if it is elliptic.

Note that if a real Lie algebra admits a non-empty open elliptic convex cone, then there exists a compactly embedded Cartan subalgebra $\mathfrak{t} \subseteq \mathfrak{g}$ (cf. [Ne99a, Th. VII.1.8]). To step further we first need some terminology concerning Lie algebras with compactly embedded Cartan subalgebras.

Definition 1.2. (cf. [Ne99a, Ch. VII]) Let $\mathfrak{g}$ be a finite dimensional Lie algebra over $\mathbb{R}$ with compactly embedded Cartan subalgebra $\mathfrak{t}$.

(a) Associated to the Cartan subalgebra $\mathfrak{t}_{\mathbb{C}}$ in the complexification $\mathfrak{g}_{\mathbb{C}}$ there is a root decomposition as follows. For a linear functional $\alpha \in \mathfrak{t}_{\mathbb{C}}^{*}$ we set

$$
\mathfrak{g}_{\mathbb{C}}^{\alpha}:=\left\{X \in \mathfrak{g}_{\mathbb{C}}:\left(\forall Y \in \mathfrak{t}_{\mathbb{C}}\right)[Y, X]=\alpha(Y) X\right\}
$$

and write $\Delta:=\left\{\alpha \in \mathfrak{t}_{\mathbb{C}}^{*} \backslash\{0\}: \mathfrak{g}_{\mathbb{C}}^{\alpha} \neq\{0\}\right\}$ for the set of roots. Then $\mathfrak{g}_{\mathbb{C}}=\mathfrak{t}_{\mathbb{C}} \oplus \bigoplus_{\alpha \in \Delta} \mathfrak{g}_{\mathbb{C}}^{\alpha}, \alpha(\mathfrak{t}) \subseteq i \mathbb{R}$ for all $\alpha \in \Delta$, and $\overline{\mathfrak{g}_{\mathbb{C}}^{\alpha}}=\mathfrak{g}_{\mathbb{C}}^{-\alpha}$, where $X \mapsto \bar{X}$ denotes complex conjugation on $\mathfrak{g}_{\mathbb{C}}$ with respect to $\mathfrak{g}$.

(b) Let $\mathfrak{r}$ denote the radical of $\mathfrak{g}$ and note that there is a $\mathfrak{t}$-invariant Levi decomposition $\mathfrak{g}=\mathfrak{r} \rtimes \mathfrak{s}$. Let $\mathfrak{k}$ be a maximal compactly embedded subalgebra of $\mathfrak{g}$ containing $\mathfrak{t}$. Then a root $\alpha$ is said to be compact if $\mathfrak{g}_{\mathbb{C}}^{\alpha} \subseteq \mathfrak{k}_{\mathbb{C}}$ and non-compact otherwise. We write $\Delta_{k}$ for the set of compact roots and $\Delta_{n}$ for the non-compact ones.

(c) A positive system $\Delta^{+}$of roots is a subset of $\Delta$ for which there exists a regular element $X_{0} \in i \mathfrak{t}^{*}$ with $\Delta^{+}:=\left\{\alpha \in \Delta: \alpha\left(X_{0}\right)>0\right\}$. We call a positive system $\mathfrak{k}$-adapted if the set 
$\Delta_{n}^{+}:=\Delta_{n} \cap \Delta^{+}$is invariant under the Weyl group $\mathcal{W}_{\mathfrak{k}}:=N_{\operatorname{Inn}_{\mathfrak{g}}(\mathfrak{k})}(\mathfrak{t}) / Z_{\operatorname{Inn}_{\mathfrak{g}}(\mathfrak{k})}(\mathfrak{t})$ acting on $\mathfrak{t}$. Recall that there exists a $\mathfrak{k}$-adapted positive system if and only if $\mathfrak{z} \mathfrak{g}(\mathfrak{z}(\mathfrak{k}))=\mathfrak{k}$. In this case we say $\mathfrak{g}$ is quasihermitian. In this case it is easy to see that $\mathfrak{s}$ is quasihermitian, too, and so all simple ideals of $\mathfrak{s}$ are either compact or hermitian.

(d) We associate to $\Delta_{n}^{+}$the convex cones

$$
C_{\min }:=\overline{\operatorname{cone}\left\{i\left[\overline{X_{\alpha}}, X_{\alpha}\right]: X_{\alpha} \in \mathfrak{g}_{\mathbb{C}}^{\alpha}, \alpha \in \Delta_{n}^{+}\right\}}
$$

and $C_{\max }:=\left(i \Delta_{n}^{+}\right)^{\star}=\left\{X \in \mathfrak{t}:\left(\forall \alpha \in \Delta_{n}^{+}\right) i \alpha(X) \geq 0\right\}$. Note that both $C_{\min }$ and $C_{\max }$ are closed convex cones in $\mathfrak{t}$.

(e) Write $p_{\mathfrak{t}}: \mathfrak{g} \rightarrow \mathfrak{t}$ for the orthogonal projection along $[\mathfrak{t}, \mathfrak{g}]$ and set $\mathcal{O}_{X}:=\operatorname{Inn}(\mathfrak{g}) . X$ for the adjoint orbit through $X \in \mathfrak{g}$. We define the minimal and maximal cone associated to $\Delta_{n}^{+}$by

$$
W_{\min }:=\left\{X \in \mathfrak{g}: p_{\mathfrak{t}}\left(\mathcal{O}_{X}\right) \subseteq C_{\min }\right\} \quad \text { and } \quad W_{\max }:=\left\{X \in \mathfrak{g}: p_{\mathfrak{t}}\left(\mathcal{O}_{X}\right) \subseteq C_{\max }\right\}
$$

and note that both cones are convex, closed and $\operatorname{Inn}(\mathfrak{g})$-invariant.

Example 1.3. (a) (Hermitian case) Let $\mathfrak{g}$ be a semisimple Lie algebra with Cartan decomposition $\mathfrak{g}=\mathfrak{k} \oplus \mathfrak{p}$. Then $\mathfrak{g}$ is called hermitian if $\mathfrak{g}$ is simple and $\mathfrak{z}(\mathfrak{k}) \neq\{0\}$. Hermitian Lie algebras are classified; the complete list is as follows (cf. [Hel78, p. 518]):

$$
\mathfrak{s u}(p, q) \quad \mathfrak{s p}(n, \mathbb{R}) \quad \mathfrak{s o}^{*}(2 n) \quad \mathfrak{s o}(2, n) \quad \mathfrak{e}_{6(-14)} \quad \mathfrak{e}_{7(-25)} .
$$

That $\mathfrak{g}$ is hermitian implies in particular that there is a compactly embedded Cartan subalgebra $\mathfrak{t} \subseteq \mathfrak{k}$ of $\mathfrak{g}$ and that $\mathfrak{z}(\mathfrak{k})=\mathbb{R} X_{0}$ is one dimensional (cf. [Hel78, Ch. VIII]). Since $X_{0}$ is $\operatorname{Inn}_{\mathfrak{g}}(\mathfrak{k})$ fixed, the prescription

$$
\Delta_{n}^{+}:=\left\{\alpha \in \Delta: \alpha\left(i X_{0}\right)>0\right\}
$$

defines a $\mathfrak{k}$-adapted system of positive non-compact roots. Hermitian Lie algebras admit pointed convex $\operatorname{Inn}(\mathfrak{g})$-invariant closed cones with non-empty elliptic interior. One of them, the minimal cone, has a quite simple description:

$$
W_{\min }=\overline{\operatorname{Inn}(\mathfrak{g}) \cdot \mathbb{R}^{+} X_{0}} .
$$

Its trace with the Cartan algebra $t$ is given by

$$
C_{\min }=\overline{\text { cone }\left\{-i \check{\alpha}: \alpha \in \Delta_{n}^{+}\right\}}
$$

where $\check{\alpha} \in i t$ denotes the coroot of $\alpha$ (cf. [HiNe93, Ch. 7] for all that).

(b) (Compact case) (cf. [HiNe93, Ch. 7]) Let $\mathfrak{k}$ be a compact semisimple Lie algebra and set $\mathfrak{g}:=\mathfrak{k} \oplus \mathbb{R}$ (direct Lie algebra sum). If $\langle\cdot, \cdot\rangle$ denotes an $\operatorname{Inn}(\mathfrak{k})$-innner product on $\mathfrak{k}$, then the prescription

$$
W:=\left\{(X, t) \in \mathfrak{g}:\langle X, X\rangle<t^{2}, t>0\right\}
$$

defines an open elliptic $\operatorname{Inn}(\mathfrak{g})$-invariant convex cone $\mathfrak{g}$. The most prominent example herof is $\mathfrak{g}=\mathfrak{u}(n)$ with

$$
W:=\{X \in \mathfrak{u}(n): i X \text { positive definite }\} .
$$

On the group level with $G=\mathrm{U}(n)$ and $G_{\mathbb{C}}=\operatorname{Gl}(n, \mathbb{C})$ the Ol'shanskiur semigroup associated to $-W$ is the unit ball in $G_{\mathbb{C}}$ :

$$
S=G \exp (-i W)=\{g \in \mathrm{Gl}(n, \mathbb{C}):\|g\|<1\}
$$


with $\|g\|$ the spectral norm.

(c) (Mixed case - The Jacobi algebra) There are interesting examples of Lie algebras admitting invariant elliptic convex cones which are neither semisimple nor solvable (see Ex. 4.9(b) for a non-nilpotent solvable example). One of them is the Jacobi algebra

$$
\mathfrak{g}:=\mathfrak{h} \mathfrak{s p}(n, \mathbb{R}):=\mathfrak{h}_{n} \rtimes \mathfrak{s p}(n, \mathbb{R})
$$

with $\mathfrak{h}_{n}=\mathbb{R} \oplus \mathbb{R}^{2 n}$ the $(2 n+1)$-dimensional Heisenberg algebra. Then $\mathfrak{z}(\mathfrak{g})=\mathfrak{z}\left(\mathfrak{h}_{n}\right) \cong \mathbb{R}$ is one dimensional and a compactly embedded Cartan subalgebra is given by $\mathfrak{t}=\mathfrak{z}(\mathfrak{g}) \oplus \mathfrak{t}_{s}$ with $\mathfrak{t}_{s}$ a compactly embedded Cartan subalgebra of $\mathfrak{s p}(n, \mathbb{R})$. For the Jacobi algebra $\mathfrak{g}$ the maximal cone has an easy description, namely

$$
W_{\max }=\mathbb{R}^{+} \oplus \mathbb{R}^{2 n} \oplus W_{\max , \mathrm{s}}
$$

with $W_{\text {max,s }}$ a maximal cone in $\mathfrak{s p}(n, \mathbb{R})$. For more details on the Jacobi algebra we refer to [KNÓ01, Sect. II] and [Ne99a, p. 400ff, p. 700]. For another mixed example see also Example 4.9 (c) below.

\section{Holomorphic representations}

Definition 1.4. Let $\mathcal{H}$ be a Hilbert space and $B(\mathcal{H})$ the space of bounded operators on it. A holomorphic representation $(\pi, \mathcal{H})$ of a complex Ol'shanskiŭ semigroup $S$ is a non-degenerate holomorphic semigroup representation $\pi: S \rightarrow B(\mathcal{H})$ such that $\pi$ is a vector-valued holomorphic map satisfying $\pi\left(s^{*}\right)=\pi(s)^{*}$ for all $s \in S$.

Now we take a closer look at the irreducible holomorphic representations of $S$. They are obtained by analytic continuation of unitary highest weight representations of $G$.

From now on we assume that $\mathfrak{g}$ contains a compactly embedded Cartan subalgebra $\mathfrak{t} \subseteq \mathfrak{g}$ and that there exists a non-empty open elliptic convex cone $W \subseteq \mathfrak{g}$. Then in view of [Ne99a, Th. VII.3.8], there exists a $\mathfrak{k}$-adapted positive system $\Delta^{+}$such that

$$
W_{\min } \subseteq \bar{W} \subseteq W_{\max }
$$

holds, $W_{\max }^{0}$ is elliptic, $W_{\min } \cap \mathfrak{t}=C_{\min }$ and $W_{\max } \cap \mathfrak{t}=C_{\max }$. Recall that every elliptic $\operatorname{Ad}(G)$ invariant cone $W \subseteq \mathfrak{g}$ can be reconstructed by its intersection with $\mathfrak{t}$, i.e., $W=\operatorname{Ad}(G) . C$ with $C:=W \cap \mathfrak{t}$.

Definition 1.5. (Highest weight modules, cf. [Ne99a, Ch. X]) Let $\Delta^{+}$be a positive system.

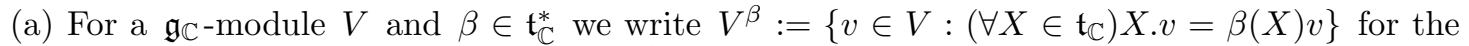
weight space of weight $\beta$ and $\mathcal{P}_{V}=\left\{\beta: V^{\beta} \neq\{0\}\right\}$ for the set of weights of $V$.

(b) Let $V$ be a $\mathfrak{g}_{\mathbb{C}}$-module and $v \in V^{\lambda}, v \neq 0$, a $\mathfrak{t}_{\mathbb{C}}$-weight vector. We say that $v$ is a primitive element of $V$ (with respect to $\Delta^{+}$) if $\mathfrak{g}_{\mathbb{C}}^{\alpha} \cdot v=\{0\}$ holds for all $\alpha \in \Delta^{+}$.

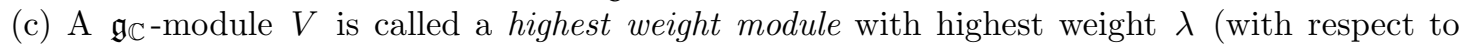
$\Delta^{+}$) if it is generated by a primitive element of weight $\lambda$.

(d) Let $G$ be a connected Lie group with Lie algebra $\mathfrak{g}$. We write $T$ for the analytic subgroup of $G$ corresponding to $\mathfrak{t}$. Let $(\pi, \mathcal{H})$ be a unitary representation of $G$. A vector $v \in \mathcal{H}$ is called $T$-finite if it is contained in a finite dimensional $T$-invariant subspace. We write $\mathcal{H}^{T, \omega}$ for the space of analytic $T$-finite vectors.

(e) An irreducible unitary representation $(\pi, \mathcal{H})$ of $G$ is called a highest weight representation with respect to $\Delta^{+}$and highest weight $\lambda \in i \mathfrak{t}^{*}$ if $\mathcal{H}^{T, \omega}$ is a highest weight module for $\mathfrak{g}_{\mathbb{C}}$ with respect to $\Delta^{+}$and highest weight $\lambda$. We write $H W\left(G, \Delta^{+}\right) \subset i t^{*}$ for the set of highest weights corresponding to unitary highest weight representations of $G$ with respect to $\Delta^{+}$. 
The interplay between irreducible holomorphic representations of $S$ and unitary highest weight representations of $G$ is described in the following lemma. Recall that a cone $W$ in a real vector space $V$ is called pointed if $W$ contains no affine lines. [St86]).

The central ideas in the following theorem go back to Ol'shanskiu (cf. [Ol82]) Stanton (cf.

Theorem 1.6. Let $S=\Gamma_{G}(W)$ be a complex Ol'shanskiน semigroup and $\Delta^{+}$be a k-adapted positive system with $C_{\min } \subseteq \bar{C} \subseteq C_{\max }$. Suppose that $W$ is pointed.

(1) If $(\pi, \mathcal{H})$ is an irreducible holomorphic representation of $S$, then $(\pi, \mathcal{H})$ extends to a strongly continuous representation of $\bar{S}$, also denoted by $(\pi, \mathcal{H})$, such that $\left.\pi\right|_{G}$ is a uniquely determined unitary highest weight representation of $G$ with respect to $\Delta^{+}$.

(2) Conversely, if $\left(\pi_{\lambda}, \mathcal{H}_{\lambda}\right)$ is a unitary highest weight representation of $G$ with respect to $\Delta^{+}$, then $\left(\pi_{\lambda}, \mathcal{H}_{\lambda}\right)$ extends to a uniquely determined strongly continuous representation of $\bar{S}$, whose restriction to $S$ is holomorphic and irreducible.

Proof. This follows from [Ne99a, Th. XI.4.8] together with its following remark.

We assume in the following that the cone $W \subseteq \mathfrak{g}$ is pointed.

Remark 1.7. In the special case of the Jacobi group

$$
G:=\operatorname{HSp}(n, \mathbb{R})=H_{n} \rtimes \operatorname{Sp}(n, \mathbb{R})
$$

(a group associated to the Jacobi algebra $\mathfrak{h} \mathfrak{s p}(n, \mathbb{R})$; cf. Example 1.3(c)) it is interesting to see what the unitary highest weight representations of $G$ are. They are given by

$$
\sigma_{\mu} \otimes \pi_{\lambda, s}
$$

where $\sigma_{\mu}$ is an extended metaplectic representation of $G$ and $\pi_{\lambda, s}$ is a unitary highest weight representation of $\operatorname{Sp}(n, \mathbb{R})$ extended trivially to the Heisenberg group $H_{n}$ (cf. [Ne99a, Sect. X.3]). All these representations extend to holomorphic representations of the maximal open complex Ol'shankiu semigroup $\Gamma_{G}\left(\operatorname{int} W_{\max }\right)$.

\section{The manifolds $\Gamma \backslash S$ and some concepts from complex analysis}

Let $S=\Gamma_{G}(W)=G \operatorname{Exp}(i W)$ be an open complex Ol'shanskil semigroup and $\Gamma<G$ a discrete subgroup. In view of the polar decomposition of $S$ (cf. Definition 1.1), the group $\Gamma$ acts via

$$
\Gamma \times S \rightarrow S, \quad(\gamma, s) \mapsto \gamma s,
$$

freely and properly discontinuously on $S$. Thus the quotient $\Gamma \backslash S$ is Hausdorff and carries a complex structure which is induced from the quotient map $S \rightarrow \Gamma \backslash S, s \mapsto \Gamma s$.

\section{Stein manifolds}

Since one of the main objectives of this paper is the investigation of the structure of the complex manifolds $\Gamma \backslash S$, especially whether they are Stein, we briefly recall here some facts concerning Stein manifolds.

If $M$ is a complex manifold, then we write $\operatorname{Hol}(M)$ for the space of holomorphic functions on $M$. We endow $\operatorname{Hol}(M)$ with the topology of compact convergence on $M$. Note that $\operatorname{Hol}(M)$ is a Fréchet space provided $M$ is second countable. 
Definition 2.1. (Stein manifolds) Let $M$ be a second countable complex manifold. Then $M$ is called a Stein manifold, if the following two axioms are satisfied

(St1) The points of $M$ are separated by $\operatorname{Hol}(M)$.

(St2) The manifold $M$ is holomorphically convex, i.e., for each compact subset $K \subseteq M$ the holomorphically convex hull

$$
\widehat{K}:=\left\{z \in M:(\forall f \in \operatorname{Hol}(M))|f(z)| \leq \sup _{w \in K}|f(w)|\right\}
$$

is compact.

Theorem 2.2. (Grauert) For a second countable complex manifold $M$, the following assertions are equivalent:

(1) The manifold $M$ is Stein.

(2) There exists a strictly plurisubharmonic exhaustion function $\varphi: M \rightarrow \mathbb{R}$, i.e., $\varphi$ is strictly plurisubharmonic and for all $r \in \mathbb{R}$ the set $\left.\left.\varphi^{-1}(]-\infty, r\right]\right)$ is a compact subset of $M$.

Proof. [Hö73, Th. 5.2.10].

Theorem 2.3. If $E \rightarrow M$ is a holomorphic principal bundle, such that the fiber and the base $M$ are Stein, then $E$ is Stein.

Proof. [MaMo60, Th. 4].

Theorem 2.4. (Neeb) Let $S$ be a complex Ol'shanskiน semigroup. Then there exists a $G \times G$ invariant strictly plurisubharmonic smooth function $\varphi: S \rightarrow \mathbb{R}^{+}$with

$$
\lim _{s_{n} \rightarrow s} \varphi\left(s_{n}\right)=\infty
$$

for all $s \in \bar{S} \backslash S$.

Proof. [Ne98, Lemma 5.11].

\section{Invariant Hilbert spaces}

We conclude this section by introducing the concept of an invariant Hilbert space of holomorphic functions.

Definition 2.5. Let $M$ be a complex manifold and $G$ a group acting on $M$ via

$$
M \times G \rightarrow M, \quad(m, g) \mapsto m . g,
$$

by holomorphic automorphisms. Then a Hilbert space $\mathcal{H} \subseteq \operatorname{Hol}(M)$ is called a $G$-invariant Hilbert space of holomorphic functions on $M$ if the following two axioms are satisfied:

(IH1) The inclusion map $\mathcal{H} \hookrightarrow \operatorname{Hol}(M)$ is continuous.

(IH2) We have a unitary representation

$$
\rho: G \rightarrow U(\mathcal{H}), \quad(\rho(g) . f)(z):=f(z . g) .
$$

Let $(\rho, \mathcal{H})$ be an invariant Hilbert space. Then by (IH1) all point evaluations $\mathcal{H} \rightarrow$ $\mathbb{C}, f \mapsto f(z)$ are continuous. Hence there exists for every $z \in M$ an element $K_{z} \in \mathcal{H}$ such that $\left\langle f, K_{z}\right\rangle=f(z)$ for all $f \in \mathcal{H}$. The function

$$
K: M \times M \rightarrow \mathbb{C}, \quad(z, w) \mapsto K(z, w):=\left\langle K_{w}, K_{z}\right\rangle,
$$

is holomorphic in the first, antiholomorphic in the second variable and $G$-invariant, i.e., we have $K(z . g, w . g)=K(z, w)$ for all $z, w \in M, g \in G$. We call the function $K$ the reproducing kernel of $(\rho, \mathcal{H})$. For further information on reproducing kernel Hilbert spaces we refer to [Ne99a, Ch. I-IV]. 


\section{Constructing $\Gamma$-spherical representations}

In this section we give a construction of $\Gamma$-spherical highest weight representations for arbitrary discrete subgroups $\Gamma<G$. It was an idea of Godement to obtain $\Gamma$-spherical representations by averaging matrix coefficients of integrable representations of $G$ (cf. [Bo66]). In our setup however it is much more convenient to use the Bergman space $\mathcal{B}^{1}(S)$ of integrable holomorphic functions on $S$ instead of $L^{1}(G)$. This is because we can naturally realize highest weight representations with sufficiently large parameter in the Hilbert space $\mathcal{B}^{2}(S)$ (cf. [Kr98a]).

\section{Hyperfunction vectors for unitary representations}

Definition 3.1. $\quad$ Let $G$ be a Lie group and $\mathcal{H}$ a Hilbert space.

(a) For a unitary representation $(\pi, \mathcal{H})$ of $G$ we denote by $\mathcal{H}^{\infty}$ and $\mathcal{H}^{\omega}$ the space of all smooth, respectively analytic vectors of $(\pi, \mathcal{H})$. The corresponding strong antiduals are denoted by $\mathcal{H}^{-\infty}$ and $\mathcal{H}^{-\omega}$ and their elements are called distribution, resp. hyperfunction vectors (see [KNÓ97, Appendix] for the definition of the topology of $\mathcal{H}^{\omega}$ ). Note that there is a natural chain of continuous inclusions

$$
\mathcal{H}^{\omega} \hookrightarrow \mathcal{H}^{\infty} \hookrightarrow \mathcal{H} \hookrightarrow \mathcal{H}^{-\infty} \hookrightarrow \mathcal{H}^{-\omega}
$$

The natural extension of $(\pi, \mathcal{H})$ to a representation on the space of hyperfunction vectors is denoted by $\left(\pi^{-\omega}, \mathcal{H}^{-\omega}\right)$ and given explicitly by

$$
\left\langle\pi^{-\omega}(g) . \nu, v\right\rangle:=\left\langle\nu, \pi\left(g^{-1}\right) \cdot v\right\rangle .
$$

Note that $\left(\pi^{-\omega}, \mathcal{H}^{-\omega}\right)$ is a continuous representation of $G$ (cf. [KNÓ97, App.]).

(b) Let $\Gamma \subseteq G$ be a closed subgroup. For a unitary representation $(\pi, \mathcal{H})$ of $G$ we write $\left(\mathcal{H}^{-\omega}\right)^{\Gamma}$ for the set of all those elements $\nu \in \mathcal{H}^{-\omega}$ satisfying $\pi^{-\omega}(\gamma) . \nu=\nu$ for all $\gamma \in \Gamma$. The unitary representation $(\pi, \mathcal{H})$ is called $\Gamma$-spherical if there exist a cyclic vector $\nu \in\left(\mathcal{H}^{-\omega}\right)^{\Gamma}$.

Remark 3.2. Even though in general the topology on the space of analytic vectors is hard to deal with, one has a quite explicit picture for holomorphic representations of complex Ol'shanskiı semigroups. Let $G$ be a connected Lie group and $(\pi, \mathcal{H})$ a unitary representation of $G$ which has an extension to a holomorphic representation of some complex Ol'shanskiu semigroup $S=$ $G \operatorname{Exp}(i W)$. Then we have for each $X \in i W$

$$
\mathcal{H}^{\omega}=\bigcup_{t>0} \pi(\operatorname{Exp}(t X)) \cdot \mathcal{H}
$$

and the topology on $\mathcal{H}^{\omega}$ is the finest locally convex topology on $\mathcal{H}^{\omega}$ making for all $t>0$ the maps $\mathcal{H} \rightarrow \mathcal{H}^{\omega}, \quad v \mapsto \pi(\operatorname{Exp}(t X)) . v$ continuous (cf. [KNÓ97, Appendix]). Note that we have $\pi(S) . \mathcal{H}^{-\omega} \subseteq \mathcal{H}^{\omega}$ and that the action of $S$ on $\mathcal{H}^{-\omega}$ is given by

$$
\left\langle\pi^{-\omega}(s) \cdot \nu, v\right\rangle:=\left\langle\nu, \pi\left(s^{*}\right) \cdot v\right\rangle .
$$

A Lie group $G$ is called a $(C A)$-Lie group $((\mathrm{CA})=$ closed adjoint) if $\operatorname{Ad}(G)$ is closed in $\operatorname{Aut}(\mathfrak{g})$. Note that most Lie groups are $(\mathrm{CA})$-Lie groups; in particular, all reductive and nilpotent Lie groups have the (CA)-property (cf. [Ne99a, VII.1.13-VII.1.15]). 
Lemma 3.3. Let $\left(\pi_{\lambda}, \mathcal{H}_{\lambda}\right)$ be a unitary highest weight representation of the (CA)-Lie group $G$ with highest weight $\lambda \in i$ int $C_{\min }^{\star}$. Then there exists a $X \in i C$ with $\operatorname{Spec}\left(d \pi_{\lambda}(X)\right) \subseteq-\beta-\mathbb{N}_{0}$ for some $\beta \in \mathbb{R}$. Let $\left(v_{n}\right)_{n \in \mathbb{N}}$ be an orthonormal basis of $\mathcal{H}_{\lambda}$ consisting of $\mathfrak{t}_{\mathbb{C}}$-weight vectors. Then for all $t>0$ there exists a constant $C_{t}>0$ such that

$$
\left(\forall w \in \mathcal{H}_{\lambda}\right) \quad \sum_{n=1}^{\infty}\left|\left\langle\pi_{\lambda}(\operatorname{Exp}(t X)) \cdot w, v_{n}\right\rangle\right| \leq C_{t}\|w\| .
$$

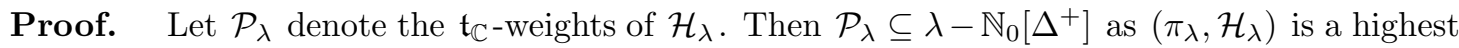
weight module with highest weight $\lambda$.

Since $C \subseteq \operatorname{int} C_{\max }$ we have $\alpha(i Y)>0$ for all $\alpha \in \Delta_{n}^{+}$and $Y \in C$. Moreover $C$ is $\mathcal{W}_{\mathfrak{k}}$-invariant so that we can find an element $Y \in C$ such that $\alpha(i Y)>0$ for all $\alpha \in \Delta^{+}$. Since $G$ is a (CA)-Lie group the group $T / Z(G)$ is compact (cf. [Ne99a, Cor. VII.1.5]) and hence we can choose $X \in i C$ such that $\alpha(i X) \in \mathbb{N}$ for all $\alpha \in \Delta^{+}$. This proves $\operatorname{Spec}\left(d \pi_{\lambda}(X)\right) \subseteq-\beta-\mathbb{N}_{0}$ for some $\beta$ : $=-\lambda(X) \in \mathbb{R}$.

Let $\mathcal{H}_{\lambda}=\bigoplus_{n=0}^{\infty} \mathcal{H}_{\lambda}^{-\beta-n}$ be the orthogonal decomposition of $\mathcal{H}_{\lambda}$ into $d \pi_{\lambda}(X)$-eigenspaces. Set $m_{n}:=\operatorname{dim} \mathcal{H}_{\lambda}^{-\beta-n}$ and note that there exists an $N \in \mathbb{N}$ such that

$$
(\forall n \in \mathbb{N}) \quad m_{n} \leq(n+1)^{N}
$$

(cf. [Ne99a, Lemma X.4.9]). Then $\left(v_{n}\right)_{n \in \mathbb{N}}=\left(v_{j}^{n}\right)_{1 \leq j \leq m_{n}, n \in \mathbb{N}}$ with $v_{j}^{n} \in \mathcal{H}_{\lambda}^{-\beta-n}$ for all $1 \leq j \leq m_{n}$.

Take now $v=\sum_{n} c_{n} v_{n}=\sum_{j, n} c_{j}^{n} v_{j}^{n}=\pi_{\lambda}(\operatorname{Exp}(t X)) . w$ for some $w \in \mathcal{H}_{\lambda}$ and $t>0$. Then $w=\sum_{j, n} e^{t(n+\beta)} c_{j}^{n} v_{j}^{n}$ and we have

$$
\begin{aligned}
\sum_{n=1}^{\infty}\left|c_{n}\right| & =\sum_{n=1}^{\infty} \sum_{1 \leq j \leq m_{n}}\left|c_{j}^{n}\right|=\sum_{n=1}^{\infty} \sum_{1 \leq j \leq m_{n}}\left|c_{j}^{n}\right| e^{t(n+\beta)} e^{-t(n+\beta)} \\
& \leq\left(\sum_{n=1}^{\infty} \sum_{1 \leq j \leq m_{n}}\left|c_{j}^{n}\right|^{2} e^{2 t(n+\beta)}\right)^{\frac{1}{2}}\left(\sum_{n=1}^{\infty} \sum_{1 \leq j \leq m_{n}} e^{-2 t(n+\beta)}\right)^{\frac{1}{2}} \\
& =\|w\|\left(\sum_{n=1}^{\infty} m_{n} e^{-2 t(n+\beta)}\right)^{\frac{1}{2}} \\
& \leq\|w\|\left(\sum_{n=1}^{\infty}(1+n)^{N} e^{-2 t(n+\beta)}\right)^{\frac{1}{2}} \leq C_{t}\|w\|
\end{aligned}
$$

with $C_{t}:=\left(\sum_{n=1}^{\infty}(1+n)^{N} e^{-2 t(n+\beta)}\right)^{\frac{1}{2}}<\infty$

\section{Bergman spaces}

If $G$ is a locally compact group, then we denote by $\mu_{G}$ a left Haar measure on $G$.

Let $S=\Gamma_{G}(W)$ be a complex Ol'shanskiu semigroup and $H<G$ a unimodular subgroup. Recall from [Kr98a, Sect. II] that there exists an $\bar{S}$-invariant positive measure $\mu_{H \backslash S}$ on $H \backslash S$. Denote by $\operatorname{Hol}(S)^{H}$ the space of left $H$-invariant holomorphic functions on $S$. For each $1 \leq$ $p \leq \infty$ we write $\|\cdot\|_{p}$ for the norm of the Banach space $L^{p}\left(H \backslash S, \mu_{H \backslash S}\right)$. For $1 \leq p \leq \infty$ we now define

$$
\mathcal{B}^{p}(H \backslash S):=\left\{f \in \operatorname{Hol}(S)^{H}:\|f\|_{p}<\infty\right\}
$$


Discrete group actions on Stein domains in complex Lie groups

and note that $\mathcal{B}^{p}(H \backslash S)$ is a Banach space of holomorphic functions on $S$ (cf. [Kr98a, Prop. II.4]); if $p=2$, then $\mathcal{B}^{2}(H \backslash S)$ is even a Hilbert space. If $H=\Gamma$ is a discrete subgroup of $G$, then we have $\operatorname{Hol}(S)^{\Gamma} \cong \operatorname{Hol}(\Gamma \backslash S)$ and we call $\mathcal{B}^{2}(\Gamma \backslash S)$ the Bergman space of the complex manifold $\Gamma \backslash S$.

Proposition 3.4. Let $S$ be a complex Ol'shanskiu semigroup and $H<G$ a unimodular subgroup. Then the following assertion holds:

(i) For all compact subsets $Q \subseteq S$ there exists a constant $C_{Q}>0$ such that

$$
\left(\forall f \in \mathcal{B}^{1}(S)\right)(\forall s \in Q) \int_{H}|f(h s)| d \mu_{H}(h) \leq C_{Q}\|f\|_{1}
$$

holds.

(ii) The linear map

$$
I^{H}: \mathcal{B}^{1}(S) \rightarrow \mathcal{B}^{1}(H \backslash S), \quad f \mapsto f^{H} ; f^{H}(s)=\int_{H} f(h s) d \mu_{H}(h),
$$

is defined and a contraction of Banach spaces.

Proof. (i) Let $Q \subseteq S$ be a compact subset. W.l.o.g. we may assume that there exists an open relatively compact subset $U \subseteq S$ such that $Q \subseteq U \subseteq \bar{U} \subseteq S$ and that $H \bar{U} \cong(H \backslash H \bar{U}) \times H$ holds. Thus we may assume that there exists a compact subset $K_{H} \subseteq H$ such that $\bar{U} \cong(H \backslash H \bar{U}) \times K_{H}$. It follows as in [Kr98a, Prop. II.4(i)] that there exists a constant $c_{Q}>0$ such that

$$
(\forall s \in Q)|f(s)| \leq c_{Q} \int_{U}|f(u)| d \mu_{S}(u)
$$

holds for all $f \in \operatorname{Hol}(S)$. For each $f \in \operatorname{Hol}(S)$ and $g \in G$ we set $f_{g}(s)=f(g s)$. Note that $f_{g} \in \mathcal{B}^{1}(S)$ provided $f \in \mathcal{B}^{1}(S)$ since $\mu_{S}$ is $G \times G$-biinvariant (cf. [Kr98a, Sect. II]). Thus we get for all $f \in \mathcal{B}^{1}(S)$ and $s \in Q$ that

$$
\begin{aligned}
\int_{H}|f(h s)| d \mu_{H}(h) & =\int_{H}\left|f_{h}(s)\right| d \mu_{H}(h) \leq c_{Q} \int_{H} \int_{U}\left|f_{h}(u)\right| d \mu_{S}(u) d \mu_{H}(h) \\
& \leq c_{Q} \int_{H} \int_{U}|f(h u)| d \mu_{S}(u) d \mu_{H}(h) \\
& =c_{Q} \int_{U} \int_{H}|f(h u)| d \mu_{H}(h) d \mu_{S}(u) \\
& =c_{Q} \int_{H \backslash H U} \int_{K_{H}} \int_{H}\left|f\left(h_{2} h_{1} u\right)\right| d \mu_{H}\left(h_{2}\right) d \mu_{H}\left(h_{1}\right) d \mu_{H \backslash S}(H u) \\
& \leq c_{Q} \mu_{H}\left(K_{H}\right) \int_{H \backslash H U} \int_{H}|f(h u)| d \mu_{H}(h) d \mu_{H \backslash S}(H u) \\
& \leq c_{Q} \mu_{H}\left(K_{H}\right) \int_{S}|f(s)| d \mu_{S}(s) \leq C_{Q}\|f\|_{1},
\end{aligned}
$$

where $C_{Q}=c_{Q} \mu_{H}\left(K_{H}\right)$. This proves (i).

(ii) Let $f \in \mathcal{B}^{1}(S)$. Then the inequality in (i) implies that $f^{H} \in \operatorname{Hol}(S)^{H}$. It remains to show that $\left\|f^{H}\right\|_{1} \leq\|f\|_{1}$. But this follows from

$$
\begin{aligned}
\left\|f^{H}\right\|_{1} & =\int_{H \backslash S}\left|f^{H}(s)\right| d \mu_{H \backslash S}=\int_{H \backslash S}\left|\int_{H} f(h s) d \mu_{H}(h)\right| d \mu_{H \backslash S}(H s) \\
& \leq \int_{H \backslash S} \int_{H}|f(h s)| d \mu_{H}(h) d \mu_{H \backslash S}(H s)=\|f\|_{1},
\end{aligned}
$$

completing the proof of (ii). 
Let $(\pi, \mathcal{H})$ be a unitary representation of a Lie group $G$ and $\left(\pi^{*}, \mathcal{H}^{*}\right)$ its dual representation. We denote by $\left(\widehat{\pi}, B_{2}(\mathcal{H})\right)$ the unitary representation of $G \times G$ on Hilbert-Schmidt operators on $\mathcal{H}$ given by $\widehat{\pi}\left(g_{1}, g_{2}\right) \cdot A=\pi\left(g_{1}\right) A \pi\left(g_{2}\right)^{-1}$ for $g_{1}, g_{2} \in G$ and $A \in B_{2}(\mathcal{H})$. Recall that the scalar product on $B_{2}(\mathcal{H})$ is given by $\langle A, B\rangle=\operatorname{tr}\left(A B^{*}\right)$ for all $A, B \in B_{2}(\mathcal{H})$. Further we have a natural equivalence

$$
\left(\pi \otimes \pi^{*}, \mathcal{H} \widehat{\otimes} \mathcal{H}^{*}\right) \rightarrow\left(\widehat{\pi}, B_{2}(\mathcal{H})\right), \quad v \otimes\langle\cdot, w\rangle \mapsto P_{v, w} ; P_{v, w}(u):=\langle u, w\rangle v .
$$

Let $(R \otimes L, \operatorname{Hol}(S))$ denote the right-left regular representation of $G \times G$ on $\operatorname{Hol}(S)$, i.e., we have

$$
\left(\forall g_{1}, g_{2} \in G\right)(\forall f \in \operatorname{Hol}(S))(\forall s \in S) \quad\left((R \otimes L)\left(g_{1}, g_{2}\right) \cdot f\right)(s)=f\left(g_{2}^{-1} s g_{1}\right) .
$$

Recall that for a unitary highest weight representation $\left(\pi_{\lambda}, \mathcal{H}_{\lambda}\right)$ all operators $\pi_{\lambda}(s), s \in S$, are of trace class (cf. [Ne99a, Th. XI.6.1]). Further the prescription

$$
\left(\widehat{\pi}_{\lambda}, B_{2}\left(\mathcal{H}_{\lambda}\right)\right) \rightarrow(R \otimes L, \operatorname{Hol}(S)), \quad A \mapsto f_{A}^{\lambda}(s)=\operatorname{tr}\left(A \pi_{\lambda}(s)\right) .
$$

gives us a $G \times G$-equivariant realization of $B_{2}\left(\mathcal{H}_{\lambda}\right)$ as $G \times G$-invariant Hilbert space of holomorphic functions on $S$ (cf. Definition 2.5)

Theorem 3.5. $\quad$ Assume that $G$ is a (CA)-Lie group and that $Z=Z(G)$ is compact. If $S$ is a complex Ol'shanskiu semigroup, then we have a $G \times G$-equivariant isomorphism of Hilbert spaces

$$
\widehat{\bigoplus}_{\lambda \in \Lambda}\left(\widehat{\pi}_{\lambda}, B_{2}\left(\mathcal{H}_{\lambda}\right)\right) \rightarrow\left(R \otimes L, \mathcal{B}^{2}(S)\right), \quad B_{2}\left(\mathcal{H}_{\lambda}\right) \ni A \mapsto \sqrt{b(\lambda)} f_{A}^{\lambda},
$$

with $b(\lambda)>0$ for all $\lambda \in \Lambda$ and

$$
\Lambda:=\left\{\lambda \in H W\left(G, \Delta^{+}\right) \cap i C^{\star}: \lambda+\rho \in i \operatorname{int} C_{\min }^{\star}, \lambda+2 \rho \in i \operatorname{int}\left(C \cap \mathfrak{t}^{+}\right)^{\star}\right\},
$$

where $\rho:=\frac{1}{2} \sum_{\alpha \in \Delta^{+}} \alpha$ and $\mathfrak{t}^{+}:=\left\{X \in \mathfrak{t}:\left(\forall \alpha \in \Delta^{+}\right) i \alpha(X) \geq 0\right\}$.

Proof. This is a special case of [Kr98a, Thm. IV.5].

For the rest of this section we assume now that $G$ is a (CA)-Lie group, $Z(G)$ is compact. We denote by $K$ the analytic subgroup of $G$ corresponding to $\mathfrak{k}$. Note that $K$ is compact by our assumptions on $G$.

Lemma 3.6. We have $2 \Lambda \subseteq \Lambda$. Further for all $\lambda \in 2 \Lambda$ and all $T$-finite analytic vectors $w \in \mathcal{H}_{\lambda}^{T, \omega}$ we have a continuous $G$-equivariant inclusion mapping

$$
\Phi: \mathcal{H}_{\lambda}^{\omega} \rightarrow \mathcal{B}^{1}(S), \quad v \mapsto f_{P_{v, w}}^{\lambda},
$$

with $f_{P_{v, w}}^{\lambda}(s)=\left\langle\pi_{\lambda}(s) \cdot v, w\right\rangle, s \in S$.

Proof. We first show that $2 \Lambda \subseteq \Lambda$. Recall the description of $\Lambda$ in Theorem 3.5. Let $\lambda \in \Lambda$. Then $\rho \in-i C_{\mathrm{min}}^{\star}$ implies that

$$
2 \lambda+\rho=2(\lambda+\rho)-\rho \in i \operatorname{int} C_{\min }^{\star}+i C_{\min }^{\star} \subseteq i \operatorname{int} C_{\min }^{\star} .
$$

Thus $2 \lambda$ is a parameter of the holomorphic discrete series and in particular we have $2 \lambda \in$ $H W\left(G, \Delta^{+}\right)$(cf. [Ne99a, Sect. XII.5] for all that). Finally we have

$$
2 \lambda+2 \rho=(\lambda+2 \rho)+\lambda \in i \operatorname{int}\left(C \cap \mathfrak{t}^{+}\right)^{\star}+i C^{\star} \subseteq i \operatorname{int}\left(C \cap \mathfrak{t}^{+}\right)^{\star}
$$


Discrete group actions on Stein domains in complex Lie groups

since $\lambda \in i C^{\star}$ and $C^{\star} \subseteq\left(C \cap \mathfrak{t}^{+}\right)^{\star}$. This concludes the proof of $2 \Lambda \subseteq \Lambda$.

Let now $\lambda \in \Lambda$ and write $v_{\lambda}$, respectively $v_{2 \lambda}$, for a normalized highest weight vector in $\mathcal{H}_{\lambda}$, respectively $\mathcal{H}_{2 \lambda}$. Denote by $\mathcal{H}_{\lambda} \widehat{\otimes} \mathcal{H}_{\lambda}$ the $G$-module where $G$ acts diagonally. Then the prescription $v_{2 \lambda} \mapsto v_{\lambda} \otimes v_{\lambda}$ gives rise to an equivariant embedding of $\mathcal{H}_{2 \lambda}$ into $\mathcal{H}_{\lambda} \widehat{\otimes} \mathcal{H}_{\lambda}$ (for more details see the proof of [HiKr99, Th. 3.2.1] where a similar situation is dealt with). In the sequel we assume that $\mathcal{H}_{2 \lambda} \subseteq \mathcal{H}_{\lambda} \widehat{\otimes} \mathcal{H}_{\lambda}$. Note that $\mathcal{H}_{2 \lambda}^{T, \omega} \subseteq \mathcal{H}_{\lambda} \otimes \mathcal{H}_{\lambda}$ so that by linearity we may assume that $w=w_{1} \otimes w_{2}$ for some $w_{1}, w_{2} \in \mathcal{H}_{\lambda}$.

Let $\left(v_{n}\right)_{n \in \mathbb{N}}$ be an orthonormal basis of $\mathcal{H}_{\lambda}$. Then $\left(v_{n} \otimes v_{m}\right)_{n, m \in \mathbb{N}}$ constitutes an orthonormal basis of $\mathcal{H}_{\lambda} \widehat{\otimes} \mathcal{H}_{\lambda}$. Hence $v \in \mathcal{H}_{2 \lambda}^{\omega}$ can be written as $v=\sum_{n, m} c_{n, m} v_{n} \otimes v_{m}$ with $\|v\|^{2}=\sum_{n, m}\left|c_{n, m}\right|^{2}$. Let $X \in i C$ be as in Lemma 3.3. Then $(X, X) \in i(C \times C)$ meets the same assumptions as $X$ in Lemma 3.3 for the unitary highest weight representation $\pi_{\lambda} \otimes \pi_{\lambda}$ of $G \times G$. Hence if $v=\pi_{2 \lambda}(\operatorname{Exp}(t X))$. $u$ for some $u \in \mathcal{H}_{2 \lambda}$ then $\sum_{n, m}\left|c_{n, m}\right| \leq C_{t}\|u\|$ by Lemma 3.3. Now the Cauchy-Schwarz inequality together with Theorem 3.5 gives

$$
\begin{aligned}
\int_{S}\left|f_{P_{v, w}}^{2 \lambda}(s)\right| & d \mu_{S}(s) \leq \sum_{n, m}\left|c_{n, m}\right| \int_{S}\left|\left\langle\left(\pi_{\lambda}(s) \otimes \pi_{\lambda}(s)\right) \cdot\left(v_{n} \otimes v_{m}\right), w_{1} \otimes w_{2}\right\rangle\right| d \mu_{S}(s) \\
& \leq \sum_{n, m}\left|c_{n, m}\right| \int_{S}\left|\left\langle\pi_{\lambda}(s) \cdot v_{n}, w_{1}\right\rangle\left\langle\pi_{\lambda}(s) \cdot v_{m}, w_{2}\right\rangle\right| d \mu_{S}(s) \\
& \leq \sum_{n, m}\left|c_{n, m}\right|\left(\int_{S}\left|\left\langle\pi_{\lambda}(s) \cdot v_{n}, w_{1}\right\rangle\right|^{2} d \mu_{S}(s)\right)^{\frac{1}{2}}\left(\int_{S}\left|\left\langle\pi_{\lambda}(s) \cdot v_{m}, w_{2}\right\rangle\right|^{2} d \mu_{S}(s)\right)^{\frac{1}{2}} \\
& =\frac{1}{b(\lambda)}\left\|w_{1}\right\| \cdot\left\|w_{2}\right\| \sum_{n, m}\left|c_{n, m}\right| \leq c_{t}\|u\|
\end{aligned}
$$

with $c_{t}:=\frac{C_{t}}{b(\lambda)}\left\|w_{1}\right\| \cdot\left\|w_{2}\right\|<\infty$. Thus we see that for all $t>0$ the mapping

$$
\mathcal{H}_{2 \lambda}^{\omega} \rightarrow \mathcal{B}^{1}(S), \quad v \mapsto \Phi(\operatorname{Exp}(t X) . v)
$$

is continuous. In view of the definition of the topology of $\mathcal{H}_{2 \lambda}^{\omega}$, this proves the continuity of $\Phi$

\section{Poincaré series}

Now we are going to construct $\Gamma$-spherical highest weight representations for generic parameters in $2 \Lambda$.

Definition 3.7. $\quad$ Let $(\pi, \mathcal{H})$ be a unitary highest weight representation for $G$. For $v \in \mathcal{H}$ we call

$$
P(v):=\sum_{\gamma \in \Gamma} \pi(\gamma) \cdot v
$$

the Poincaré series of $v$, provided $P(v)$ exists in $\mathcal{H}^{-\omega}$, i.e., if

$$
\mathcal{H}^{\omega} \rightarrow \mathbb{C}, \quad w \mapsto \sum_{\gamma \in \Gamma}\langle\pi(\gamma) \cdot v, w\rangle
$$

is a continuous antilinear functional. Note that $P(v) \in\left(\mathcal{H}^{-\omega}\right)^{\Gamma}$. 
Proposition 3.8. Let $\left(\pi_{\lambda}, \mathcal{H}_{\lambda}\right)$ be a unitary highest weight representation of $G$ with $\lambda \in 2 \Lambda$. Then for all $w \in \mathcal{H}_{\lambda}^{T, \omega}$ the Poincaré series $P(w)$ exists, and defines a $\Gamma$-fixed hyperfunction vector. Moreover, for all $v \in \mathcal{H}_{\lambda}^{\omega}$ the series $\langle P(w), v\rangle=\sum_{\gamma \in \Gamma}\langle\pi(\gamma) . w, v\rangle$ converges absolutely.

Proof. $\quad$ Let $w \in \mathcal{H}_{\lambda}^{T, \omega}, v \in \mathcal{H}_{\lambda}^{\omega}$ and set

$$
f_{v}: \bar{S} \rightarrow \mathbb{C}, \quad s \mapsto\left\langle\pi_{\lambda}(s) \cdot v, w\right\rangle .
$$

By Lemma 3.6 we have $f_{v} \in \mathcal{B}^{1}(S)$ and so $f_{v}^{\Gamma} \in \mathcal{B}^{1}(\Gamma \backslash S)$ with $f_{v}^{\Gamma}$ converging absolutely on all compact subsets $Q \subseteq S$, i.e.,

$$
\left(\exists C_{Q}>0\right)(\forall s \in Q)\left(\forall f \in \mathcal{B}^{1}(S)\right) \quad \sum_{\gamma \in \Gamma}|f(\gamma s)| \leq C_{Q}\|f\|_{1}
$$

(cf. Proposition 3.4). Let $X \in i C$ and set $s_{t}:=\operatorname{Exp}(t X)$ for all $t>0$. Then Lemma 3.6 asserts in particular that for all $t>0$ there exists a constant $C_{t}>0$ such that

$$
\left(\forall v \in \mathcal{H}_{\lambda}\right) \quad\left\|f_{\pi_{\lambda}\left(s_{t}\right) . v}\right\|_{1} \leq C_{t}\|v\| .
$$

By the definition of the topology on $\mathcal{H}_{\lambda}^{\omega}$, we only have to check the continuity of the maps

$$
\mathcal{H}_{\lambda} \rightarrow \mathbb{C}, \quad v \mapsto\left\langle\pi_{\lambda}\left(s_{t}\right) . v, P(w)\right\rangle .
$$

Fix now $t>0$ and choose $Q$ such that $s:=s_{\frac{t}{2}} \in Q$. Then (3.1) and (3.2) imply that

$$
\begin{aligned}
\left|\left\langle\pi_{\lambda}\left(s_{t}\right) \cdot v, P(w)\right\rangle\right| & =\left|\sum_{\gamma \in \Gamma}\left\langle\pi_{\lambda}\left(s_{t}\right) \cdot v, \pi_{\lambda}(\gamma) \cdot w\right\rangle\right| \\
& \leq \sum_{\gamma \in \Gamma}\left|\left\langle\pi_{\lambda}(s) \pi_{\lambda}(s) \cdot v, \pi_{\lambda}(\gamma) \cdot w\right\rangle\right|=\sum_{\gamma \in \Gamma}\left|f_{\pi_{\lambda}(s) \cdot v}\left(\gamma^{-1} s\right)\right| \\
& \leq C_{Q}\left\|f_{\pi_{\lambda}(s) \cdot v}\right\|_{1} \leq C_{Q} C_{\frac{t}{2}}\|v\| .
\end{aligned}
$$

This proves the proposition.

Now we are going to show that the Poincaré series of the highest weight vector $P\left(v_{\lambda}\right)$ is non-zero for almost all parameters $\lambda \in 2 \Lambda$. For that we have to recall some facts concernig the generalized Harish-Chandra decomposition. Our source of reference is [Ne99a, Sect. XII.1].

We denote by $K$ the analytic subgroup of $G$ corresponding to $\mathfrak{k}$. Note that $K$ is compact by our assumptions on $G$. Let $K_{\mathbb{C}}$ denote the universal complexification of $K$ and note that $K \subseteq K_{\mathbb{C}}$. By $G_{\mathbb{C}}$ we denote the universal complexification of $G$.

We define subalgebras of $\mathfrak{g}_{\mathbb{C}}$ by

$$
\mathfrak{p}^{+}:=\oplus_{\alpha \in \Delta_{n}^{+}} \mathfrak{g}_{\mathbb{C}}^{\alpha} \quad \text { and } \quad \mathfrak{p}^{-}:=\oplus_{\alpha \in \Delta_{n}^{-}} \mathfrak{g}_{\mathbb{C}}^{\alpha}
$$

and note that $\mathfrak{p}^{ \pm}$are abelian since $W$ was assumed to be pointed. Further we have $\mathfrak{g}_{\mathbb{C}}=$ $\mathfrak{p}^{-} \oplus \mathfrak{k}_{\mathbb{C}} \oplus \mathfrak{p}^{+}$.

Let $P^{ \pm}$denote the analytic subgroups of $G_{\mathbb{C}}$ corresponding to $\mathfrak{p}^{ \pm}$. Assume for the moment that $G \subseteq G_{\mathbb{C}}$. Then the multiplication mapping

$$
P^{-} \times K_{\mathbb{C}} \times P^{+} \rightarrow P^{-} K_{\mathbb{C}} P^{+}, \quad\left(p_{-}, k, p_{+}\right) \mapsto p_{-} k p_{+} .
$$

is biholomorphic and we have $G \subseteq \bar{S} \hookrightarrow P^{-} K_{\mathbb{C}} P^{+}$.

For the general case we can use some standard covering theory to lift the results from above: We obtain a complex manifold $P^{-} K_{\mathbb{C}} P^{+}$with a biholomorphic map $P^{-} \times K_{\mathbb{C}} \times P^{+} \rightarrow P^{-} K_{\mathbb{C}} P^{+}$ and the inclusion chain from before also lifts: $G \subseteq \bar{S} \hookrightarrow P^{-} K_{\mathbb{C}} P^{+}$.

We denote by $\kappa: \bar{S} \rightarrow K_{\mathbb{C}}$ the middle projection. Set $\mathfrak{t}_{0}=\mathfrak{t} \cap[\mathfrak{k}, \mathfrak{k}]$ and note that $\mathfrak{t}=\mathfrak{t}_{0} \oplus \mathfrak{z}(\mathfrak{k})$. Accordingly we have $\mathfrak{t}^{*}=\mathfrak{t}_{0}^{*} \oplus \mathfrak{z}(\mathfrak{k})^{*}$. 
Discrete group actions on Stein domains in complex Lie groups

Proposition 3.9. Let $\lambda_{0} \in i t_{0}^{*}$ be dominant integral for $\Delta_{k}^{+}$.

(i) There exists a lattice $\Gamma_{\mathfrak{z}(\mathfrak{k})} \subseteq i \mathfrak{z}(\mathfrak{k})^{*}$ such that

$$
\lambda_{0}+\left(\Gamma_{\mathfrak{z}(\mathfrak{k})} \cap i C^{\star}\right) \backslash\{0\} \subseteq 2 \Lambda .
$$

(ii) Fix $\zeta \in H W\left(G, \Delta^{+}\right) \cap\left(\left(\Gamma_{\mathfrak{z}(\mathfrak{k})} \cap i C^{\star}\right) \backslash\{0\}\right)$ and set $\lambda_{n}:=\lambda_{0}+n \zeta$. Then there exists an $N_{0} \in \mathbb{N}$ such that $\lambda_{n} \in 2 \Lambda$ for all $n \geq N_{0}, n \in \mathbb{N}$. Further there exists an $n \in \mathbb{N}, n \geq N_{0}$ such that

$$
\left\langle P\left(v_{\lambda_{n}}\right), v_{\lambda_{n}}\right\rangle \neq 0
$$

where $v_{\lambda_{n}}$ is a normalized highest weight vector for $\left(\pi_{\lambda_{n}}, \mathcal{H}_{\lambda_{n}}\right)$.

Proof. (i) This follows from the structure of the set of unitary highest weights (cf. [Kr99, Sect. IV]) together with our explicit description of $\Lambda$.

(ii) From the definition of $\Lambda$ it is clear that there exists an $N_{0} \in \mathbb{N}$ such that $\lambda_{n} \in 2 \Lambda$ for all $n \geq N_{0}, n \in \mathbb{N}$. Define $\mathbb{N}_{\geq N_{0}}:=\mathbb{N} \cap\left[N_{0}, \infty[\right.$. We consider the function

$$
F: \mathbb{N}_{\geq N_{0}} \rightarrow \mathbb{C}, \quad n \mapsto\left\langle P\left(v_{\lambda_{n}}\right), v_{\lambda_{n}}\right\rangle=\sum_{\gamma \in \Gamma}\left\langle\pi_{\lambda_{n}}(\gamma) \cdot v_{\lambda_{n}}, v_{\lambda_{n}}\right\rangle
$$

Note that the defining series converges absolutely by Proposition 3.8.

If $\left(\pi_{\lambda}, \mathcal{H}_{\lambda}\right)$ is any unitary highest weight representation of $G$, then $F(\lambda):=\operatorname{span}\left\{\pi(K) \cdot v_{\lambda}\right\}$ is a finite dimensional irreducible representation of $K$. Hence the representation $\left(\pi_{\lambda}, F(\lambda)\right)$ of $K$ naturally extends to a holomorphic representation of $K_{\mathbb{C}}$. By [Kr98b, Prop. II.20] we now have $\left\langle\pi_{\lambda}(s) \cdot v_{\lambda}, v_{\lambda}\right\rangle=\left\langle\pi_{\lambda}(\kappa(s)) \cdot v_{\lambda}, v_{\lambda}\right\rangle$ for all $\lambda \in H W\left(G, \Delta^{+}\right)$and $s \in \bar{S}$, and so

$$
(\forall n \in \mathbb{N}) \quad F(n)=\sum_{\gamma \in \Gamma}\left\langle\pi_{\lambda_{n}}(\kappa(\gamma)) \cdot v_{\lambda_{n}}, v_{\lambda_{n}}\right\rangle .
$$

Let $\mathfrak{n}_{k}:=\bigoplus_{\alpha \in \Delta_{k}^{+}} \mathfrak{g}_{\mathbb{C}}^{\alpha}, \overline{\mathfrak{n}_{k}}:=\bigoplus_{\alpha \in \Delta_{k}^{-}} \mathfrak{g}_{\mathbb{C}}^{\alpha}$ be the complex conjugate and $N_{k}, \overline{N_{k}}$, the respective analytic subgroups of $K_{\mathbb{C}}$. Then we have the Bruhat decomposition

$$
K_{\mathbb{C}}=\bigcup_{w \in \mathcal{W}_{\mathfrak{k}}} \overline{N_{k}} \widetilde{w} T_{\mathbb{C}} N_{k},
$$

where $\widetilde{w} \in \widetilde{K}_{\mathbb{C}}$ represents $w$. Fix $n \in \mathbb{N}$ and consider the function

$$
f: K_{\mathbb{C}} \rightarrow \mathbb{C}, \quad k \mapsto\left\langle\pi_{\lambda_{n}}(k) \cdot v_{\lambda_{n}}, v_{\lambda_{n}}\right\rangle .
$$

The fact that $\lambda_{0}$ is regular implies that $f$ vanishes on all cells $\overline{N_{k}} \widetilde{w} T_{\mathbb{C}} N_{k}$ with $\widetilde{w} \neq \mathbf{1}$. Write an element $k \in \overline{N_{k}} T_{\mathbb{C}} N_{k}$ as $k=\bar{n}(k) t(k) n(k)$ and note that $f(k)=f(t(k))=t(k)^{\lambda_{n}}$. Thus we have

$$
\left(\forall k \in K_{\mathbb{C}}\right) \quad f(k)= \begin{cases}0 & \text { if } k \notin \overline{N_{k}} T_{\mathbb{C}} N_{k} \\ t(k)^{\lambda_{n}} & \text { if } k \in \overline{N_{k}} T_{\mathbb{C}} N_{k}\end{cases}
$$

The set $\left\{t(\kappa(\gamma)) \in T_{\mathbb{C}}: \gamma \in \Gamma\right\}$ defines a countable family $\left(t_{j}\right)_{j \in \mathbb{N}} \subseteq T_{\mathbb{C}}$. Then (3.3) and (3.4) give that

$$
F: \mathbb{N}_{\geq N_{0}} \rightarrow \mathbb{C}, \quad n \mapsto \sum_{j=1}^{\infty} t_{j}^{n\left(\lambda_{0}+\zeta\right)}
$$

Now it follows from Lemma 3.10 below that $F(n) \neq 0$ for at least one $n \in \mathbb{N}$ proving the proposition. 
Lemma 3.10. Let $V$ be a finite dimensional real vector space, $V^{*}$ its dual and $V_{\mathbb{C}}=V+i V$ its complexification. Let $C \subseteq V^{*}$ be a convex cone and $\Gamma \subseteq V^{*}$ be a lattice. Suppose that $C \cap \Gamma \neq \varnothing$ and that there exists a sequence $\left(z_{n}\right)_{n \in \mathbb{N}}$ in $V_{\mathbb{C}}$ such that

$$
F: C \cap \Gamma \rightarrow \mathbb{C}, \quad \lambda \mapsto \sum_{n \in \mathbb{N}} e^{\lambda\left(z_{n}\right)},
$$

is defined by absolutely convergent series. Then $F \neq 0$.

Proof. The assertion of the lemma easily reduces to the one dimensional case, i.e., $V=\mathbb{R}$. By rescaling we may assume that $\Gamma=\mathbb{Z}$. Then $C=\mathbb{R}$ or $C= \pm] 0, \infty[$ and taking a subcone of $C$ we, up to sign, may assume that $C=] 0, \infty[$. Then we have $\mathbb{Z} \cap] 0, \infty[=\mathbb{N}$ and

$$
F: \mathbb{N} \rightarrow \mathbb{C}, \quad m \mapsto \sum_{n=1}^{\infty} e^{m z_{n}}
$$

with $\sum_{n=1}^{\infty} e^{m \operatorname{Re}\left(z_{n}\right)}<\infty$ for all $m \in \mathbb{N}$. Hence $\operatorname{Re}\left(z_{n}\right)>0$ for only finitely many $n \in \mathbb{N}$ and

$$
x_{0}:=\sup _{n \in \mathbb{N}}\left\{\operatorname{Re} z_{n}: n \in \mathbb{N}\right\}<\infty .
$$

Further $F$ extends to a bounded analytic function

$$
f:] 1, \infty\left[\rightarrow \mathbb{C}, \quad \lambda \mapsto \sum_{n=1}^{\infty} e^{\lambda z_{n}} .\right.
$$

To obtain a contradiction, assume that $F=0$. Then $f=0$ by [Kr01, App. A].

W.l.o.g. we may assume that $\operatorname{Re} z_{n}=x_{0}$ exactly for $1 \leq n \leq N$ for some $N \in \mathbb{N}$ and that $z_{1}=z_{j}$ exactly for $1 \leq j \leq k, k \leq N$. Then dominated convergence gives that

$$
\lim _{\lambda \rightarrow \infty} e^{-\lambda z_{1}}\left(\sum_{j=N+1}^{\infty} e^{\lambda z_{j}}\right)=0 .
$$

Choose $\lambda_{0}>0$ such that $\left|e^{-\lambda z_{1}}\left(\sum_{j=N+1}^{\infty} e^{\lambda z_{j}}\right)\right|<\frac{1}{2}$ for all $\lambda>\lambda_{0}$. Then $f=0$ implies that

$$
\left(\forall \lambda>\lambda_{0}\right) \quad\left|k+\sum_{j=k+1}^{N} e^{i \lambda \operatorname{Im}\left(z_{j}-z_{1}\right)}\right|<\frac{1}{2} .
$$

Since the one parameter subsemigroup

$$
\left\{\left(e^{i \lambda \operatorname{Im}\left(z_{k+1}-z_{1}\right)}, \ldots, e^{i \lambda \operatorname{Im}\left(z_{N}-z_{1}\right)}\right): \lambda>\lambda_{0}\right\}
$$

in $\left(\mathrm{S}^{1}\right)^{N-k}$ contains elements arbitary close to the identity $\mathbf{1} \in\left(\mathrm{S}^{1}\right)^{N-k}$, we arrive at a contradiction, proving the lemma.

We now summarize the main results of this section.

Theorem 3.11. Let $S=\Gamma_{G}(W)$ be a complex Ol'shanskiน semigroup with $G$ a (CA)-Lie group and $Z(G)$ compact. Let $U$ be any open cone in $i C^{\star}$. Then for every discrete subgroup $\Gamma<G$ there exist a $\Gamma$-spherical contractive unitary highest weight representation $\left(\pi_{\lambda}, \mathcal{H}_{\lambda}\right)$ of $G$ with $\lambda \in U$ and compact kernel.

Proof. Note that non-zero $\Gamma$-fixed hyperfunction vectors for a unitary highest weight representation $\left(\pi_{\lambda}, \mathcal{H}_{\lambda}\right)$ are cyclic, since $\mathcal{H}_{\lambda}^{T, \omega}$ is dense in $\mathcal{H}_{\lambda}^{\omega}$ (cf. [HiKr99, Lemma 6.2.1]). Therefore it follows from Proposition 3.8 and Proposition 3.9 (ii) that there exists a $\Gamma$-spherical unitary highest weight representation of $G$. Moreover the representations belonging to a parameter $\lambda \in 2 \Lambda$ are contractive since $\Lambda \subseteq i C^{\star}$ (cf. [Kr99, Lemma IV.13]). The remaining statement of the theorem now follows from Proposition 3.9(ii) together with [Ne99a, Lemma X.4.7, Th. X.4.8]. 


\section{Point separation and vansihing at infinity}

In this section we construct rich classes of holomorphic functions on $\Gamma \backslash S$. For $\Gamma<G$ cocompact we construct a class of point separating holomorphic functions on $\Gamma \backslash S$ which vanish at infinity. For arbitray $\Gamma$ we will show that $\operatorname{Hol}(\Gamma \backslash S)$ separates the points.

\section{Compact $G$-orbits in the hyperfunctions}

Lemma 4.1. Let $s_{0}=\operatorname{Exp}\left(X_{0}\right)$ with $X_{0} \in i C$. Then the following assertions hold:

(i) $S s_{0} \subseteq G \operatorname{Exp}\left(\operatorname{conv}\left(\mathcal{W}_{\mathfrak{k}} \cdot X_{0}\right)+i C\right) G$.

(ii) There exists an $X \in i C$ such that

$$
S s_{0} \subseteq G \operatorname{Exp}(X+i C) G
$$

Proof. (i) For $Z \in i C$ set $F_{Z, X_{0}}:=\operatorname{conv}\left(\mathcal{W}_{\mathfrak{k}} \cdot\left(Z+X_{0}\right)\right)+i C$. Then [Ne97, Prop. V.1] shows that

$$
(\forall Z \in i C) \quad \operatorname{Exp}(Z) G \operatorname{Exp}\left(X_{0}\right) \subseteq G \operatorname{Exp}\left(F_{Z, X_{0}}\right) G
$$

Now we have

$$
\mathcal{W}_{\mathfrak{k}} \cdot\left(Z+X_{0}\right) \subseteq \mathcal{W}_{\mathfrak{k}} \cdot Z+\mathcal{W}_{\mathfrak{k}} \cdot X_{0} \subseteq \mathcal{W}_{\mathfrak{k}} \cdot X_{0}+i C \subseteq \operatorname{conv}\left(\mathcal{W}_{\mathfrak{k}} \cdot X_{0}\right)+i C
$$

for all $Z \in i C$. Hence (i) follows from $S=G \operatorname{Exp}(i C) G$.

(ii) Let $\leq_{C}$ denote the conal order on the convex cone $i C$, i.e., for $X, Y \in i C$ one has $X \leq_{C} Y$ if and only if $X \in Y+i C$. Since $\operatorname{conv}\left(\mathcal{W}_{\mathfrak{k}} \cdot X_{0}\right)$ is compact, we therefore find an element $Y \in i C$ such that $Y \leq_{C} \operatorname{conv}\left(\mathcal{W}_{\mathfrak{k}} \cdot X_{0}\right)$, i.e., $\operatorname{conv}\left(\mathcal{W}_{\mathfrak{k}} \cdot X_{0}\right) \subseteq Y+i C$. In view of (i), this proves (ii).

Lemma 4.2. Let $(\pi, \mathcal{H})$ be a holomorphic representation of $S$. Suppose that $\Gamma \backslash G$ is compact and let $v^{\Gamma} \in\left(\mathcal{H}^{-\omega}\right)^{\Gamma}$. Then the following assertions hold:

(i) The orbit $\pi^{-\omega}(G) \cdot v^{\Gamma}$ is compact in $\mathcal{H}^{-\omega}$.

(ii) For all $X_{0} \in i C$ the set $\pi^{-\omega}\left(\operatorname{Exp}\left(X_{0}\right)\right) \pi^{-\omega}(G) \cdot v^{\Gamma}$ is compact in $\mathcal{H}$.

Proof. (i) Recall from Definition 3.1 that the representation $\left(\pi^{-\omega}, \mathcal{H}^{-\omega}\right)$ of $G$ is continuous, i.e., the map

$$
G \times \mathcal{H}^{-\omega} \rightarrow \mathcal{H}^{-\omega}, \quad(g, v) \mapsto \pi^{-\omega}(g) \cdot v,
$$

is continuous. Thus $\pi^{-\omega}(G) \cdot v^{\Gamma}$ is compact by the cocompactness of $\Gamma$ in $G$.

(ii) Recall the definition of the topology on $\mathcal{H}^{\omega}$ (cf. Remark 3.2). Being the strong dual of the analytic vectors $\mathcal{H}^{\omega}$, the topology on $\mathcal{H}^{-\omega}$ is the finest locally convex topology for which all maps

$$
\mathcal{H}^{-\omega} \rightarrow \mathcal{H}, \quad v \mapsto \pi^{-\omega}\left(\operatorname{Exp}\left(t X_{0}\right)\right) \cdot v, \quad t>0,
$$

become continuous. Hence for all compact subsets $K \subseteq \mathcal{H}^{-\omega}$, the set $\pi^{-\omega}\left(\operatorname{Exp}\left(X_{0}\right)\right) . K$ is compact in $\mathcal{H}$. In view of (i), this proves (ii). 


\section{Realizing $\Gamma$-spherical representations in holomorphic functions}

Throughout this subsection $(\pi, \mathcal{H})$ denotes a holomorphic representation of a complex Ol'shanskii semigroup. We assume that $(\pi, \mathcal{H})$ is $\Gamma$-spherical for an arbitrary discrete subgroup $\Gamma<G$.

Fix now a cyclic element $v^{\Gamma} \in\left(\mathcal{H}^{-\omega}\right)^{\Gamma}$. By [KNÓ97, App.] the mapping

$$
r: \mathcal{H} \rightarrow \operatorname{Hol}(\Gamma \backslash S), \quad v \mapsto f_{v} ; f_{v}(\Gamma s):=\left\langle\pi(s) . v, v^{\Gamma}\right\rangle,
$$

is injective, continuous and $G$-equivariant, i.e., the map $r$ gives us a realization of $\mathcal{H}$ as a $G$ invariant Hilbert space of holomorphic functions on $\Gamma \backslash S$ (cf. Definition 2.5). In the sequel we assume that $\mathcal{H} \subseteq \operatorname{Hol}(\Gamma \backslash S)$. Recall from Section 2 that there is a reproducing kernel $K$ for $\mathcal{H}$.

Lemma 4.3. Let $(\pi, \mathcal{H})$ be a $\Gamma$-spherical holomorphic representation of $S$ realized in the holomorphic functions on $\Gamma \backslash S$ via the realization (4.1). If $K$ is the reproducing kernel of $\mathcal{H}$, then $K_{\Gamma s}=\pi\left(s^{*}\right) \cdot v^{\Gamma}, s \in S$, and

$$
(\forall s, t \in S) \quad K(\Gamma s, \Gamma t)=\left\langle\pi\left(t^{*}\right) \cdot v^{\Gamma}, \pi\left(s^{*}\right) \cdot v^{\Gamma}\right\rangle .
$$

Proof. This is analogous to the proof of [HiKr01, Th. 4.1.1].

Proposition 4.4. Assume that $\Gamma \backslash G$ is compact and let $(\pi, \mathcal{H})$ be a $\Gamma$-spherical holomorphic contraction representation of $S$. Then the following assertions hold:

(i) Fix $X_{0} \in i C$ and set $s_{0}:=\operatorname{Exp}\left(X_{0}\right)$. Then, the reproducing kernel $K$ of $\mathcal{H}$ is bounded when restricted to $\Gamma \backslash S s_{0} \times \Gamma \backslash S s_{0}$. In particular, there exists a constant $C=C\left(s_{0}\right)$ such that

$$
\left(\forall s \in S s_{0}\right) \quad\left\|K_{\Gamma s}\right\| \leq C .
$$

(ii) All analytic vectors $\mathcal{H}^{\omega}$ are bounded holomorphic functions on $\Gamma \backslash S$.

Proof. (i) By Lemma 4.3 we have $K(\Gamma s, \Gamma t)=\left\langle\pi\left(t^{*}\right) \cdot v^{\Gamma}, \pi\left(s^{*}\right) \cdot v^{\Gamma}\right\rangle$. Since $|K(z, w)|^{2} \leq$ $K(z, z) K(w, w)$ for all $z, w \in \Gamma \backslash S$ (cf. [Ne99a, Ch. I]), we have

$$
\sup _{z, w \in \Gamma \backslash S}|K(z, w)| \leq \sup _{z \in \Gamma \backslash S} K(z, z) .
$$

In view of Lemma 4.1 we have $S s_{0} \subseteq G \operatorname{Exp}(X) \operatorname{Exp}(i C) G$ for some $X \in i C$. Therefore we get for all $s \in S$ that

$$
\left\|K_{\Gamma s s_{0}}\right\|^{2}=K\left(\Gamma s s_{0}, \Gamma s s_{0}\right)=\left\|\pi\left(s s_{0}\right) \cdot v^{\Gamma}\right\|^{2} \leq \sup _{g \in G}\left\|\pi(\operatorname{Exp}(X)) \pi(g) \cdot v^{\Gamma}\right\|^{2},
$$

since $(\pi, \mathcal{H})$ is contractive and $\operatorname{Exp}(X)$ commutes with $\operatorname{Exp}(i C)$. But now the assertion follows from Lemma 4.2(ii).

(ii) Let $f \in \mathcal{H}^{\omega}$. Then $f \in \pi\left(s_{0}\right)$. H for some $s_{0}=\operatorname{Exp}\left(X_{0}\right), X_{0} \in i C$. Thus we can write $f=\pi\left(s_{0}\right) . g$ with $g \in \mathcal{H}$. But then (i) implies that

$$
\sup _{s \in S}|f(\Gamma s)|=\sup _{s \in S s_{0}}|g(s)|=\sup _{s \in S s_{0}}\left|\left\langle g, K_{\Gamma s}\right\rangle\right| \leq C\left(s_{0}\right)\|g\| .
$$




\section{Vanishing at infinity}

In this subsection we show that the analytic vectors of certain invariant Hilbert spaces of holomorphic functions on $\Gamma \backslash S$ are vanishing at infinity.

Lemma 4.5. Let $s_{0}=\operatorname{Exp}\left(X_{0}\right) \in S, X_{0} \in i C$, and $\left(s_{n}\right)_{n \in \mathbb{N}}$ be a sequence in $S s_{0}$.

(i) There exists an element $X \in i C$ such that

$$
s_{n}=g_{n} h_{n}^{-1} \operatorname{Exp}\left(X+X_{n}\right) h_{n}
$$

with $g_{n}, h_{n} \in G, X_{n} \in i C$.

(ii) Assume now that $\Gamma<G$ is cocompact and that $\left(\Gamma s_{n}\right)_{n \in \mathbb{N}}$ leaves every compact subset in $\Gamma \backslash \bar{S}$. Then the sequence $h_{n}^{-1} \operatorname{Exp}\left(X+X_{n}\right) h_{n}$ leaves every compact subset of $\operatorname{Exp}(\bar{W})$. In particular we have $X_{n} \rightarrow \infty$ in $i \bar{C}$ or $h_{n} \rightarrow \infty$ in $G$.

Proof. (i) This follows from Lemma 4.1.

(ii) Note that there is a polar decomposition of $\Gamma \backslash \bar{S}$

$$
\Gamma \backslash G \times \bar{W} \rightarrow \Gamma \backslash \bar{S}
$$

which is a homeomorphic mapping. As $\Gamma \backslash G$ is compact, (ii) follows now from (i).

Theorem 4.6. (Vanishing at Infinity) Let $\Gamma<G$ be cocompact and $\left(\pi_{\lambda}, \mathcal{H}_{\lambda}\right)$ be a $\Gamma$-spherical unitary highest weight representation of $G$ with $\lambda \in i$ int $C^{\star}$. Assume further that $G$ is a (CA)Lie group and that $Z(G)$ is compact. Let $v \in \mathcal{H}^{\omega}$ and $f_{v}(\Gamma s)=\left\langle\pi(s) . v, v^{\Gamma}\right\rangle$ the corresponding holomorphic function on $\Gamma \backslash S$. Then $f_{v}$ extends to a continuous function on $\Gamma \backslash \bar{S}$, also denoted by $f_{v}$ and the following assertions hold:

(i) The function $f_{v}$ is bounded.

(ii) We have $\lim _{\substack{s \rightarrow \infty \backslash \bar{S} \\ s \in \Gamma}} f_{v}(s)=0$.

Proof. Fix $X_{0} \in i C$ and set $s_{t}:=\operatorname{Exp}\left(t X_{0}\right)$ for $t>0$. Then $\mathcal{H}_{\lambda}^{\omega}=\cup_{t>0} \pi_{\lambda}\left(s_{t}\right)$. $\mathcal{H}_{\lambda}$ shows that there is a $t_{0}>0$ such that $v=\pi_{\lambda}\left(s_{t_{0}}\right)$.w for some $w \in \mathcal{H}_{\lambda}$. Set $s_{0}:=s_{t_{0}}$. Then $\bar{S} s_{0} \subseteq S$ shows that there is a continuous extension of $f_{v}$ to $\Gamma \backslash \bar{S}$.

(i) Proposition 4.4(ii).

(ii) Let $\left(s_{n}\right)_{n \in \mathbb{N}}$ be a sequence in $S$ such that $\Gamma s_{n} \rightarrow \infty$ in $\Gamma \backslash \bar{S}$. We claim that $\Gamma s_{n} s_{0} \rightarrow \infty$ in $\Gamma \backslash \bar{S}$. For that it is sufficient to show that the mapping

$$
\rho_{\Gamma}\left(s_{0}\right): \Gamma \backslash \bar{S} \rightarrow \Gamma \backslash \overline{;} \Gamma \mapsto \Gamma s_{0}
$$

is proper. Recall from [HiNe93, Th. 3.20] that $\bar{S}$ acts via right multiplication on $\bar{S}$ by proper maps. In particular, $\rho\left(s_{0}\right): \bar{S} \rightarrow \bar{S}, s \mapsto s s_{0}$ is proper. Let now $Q_{\Gamma} \subseteq \Gamma \backslash \bar{S}$ be a compact subset. Then we find a compact subset $Q \subseteq \bar{S}$ such that $Q_{\Gamma}=\Gamma \backslash \Gamma Q$. Since $\rho\left(s_{0}\right)$ is proper, the set $\rho\left(s_{0}\right)^{-1}(Q)$ is compact. Hence $\rho_{\Gamma}\left(s_{0}\right)^{-1}\left(Q_{\Gamma}\right)=\Gamma \backslash \Gamma \rho\left(s_{0}\right)^{-1}(Q)$ is compact, establishing our claim.

By Lemma 4.5 we find an element $X \in C$ such that

$$
s_{n} s_{0}=g_{n} h_{n}^{-1} \operatorname{Exp}\left(X+X_{n}\right) h_{n}
$$

for elements $g_{n}, h_{n} \in G, X_{n} \in i C$. Then Lemma 4.3 and Proposition 4.4 show that

$$
\begin{aligned}
f_{v}\left(\Gamma s_{n}\right) & =f_{w}\left(\Gamma s_{n} s_{0}\right)=\left\langle w, K_{\Gamma s_{n} s_{0}}\right\rangle=\left\langle w, \pi_{\lambda}\left(s_{0} s_{n}^{*}\right) \cdot v^{\Gamma}\right\rangle \\
& =\left\langle w, \pi_{\lambda}\left(h_{n}^{-1} \operatorname{Exp}\left(X+X_{n}\right)\right) \pi_{\lambda}^{-\omega}\left(h_{n} g_{n}^{-1}\right) \cdot v^{\Gamma}\right\rangle \\
& =\left\langle\pi_{\lambda}\left(h_{n}\right) \cdot w, \pi_{\lambda}\left(\operatorname{Exp}\left(\frac{1}{2} X+X_{n}\right)\right) \pi_{\lambda}^{-\omega}\left(\operatorname{Exp}\left(\frac{1}{2} X\right) h_{n} g_{n}^{-1}\right) \cdot v^{\Gamma}\right\rangle .
\end{aligned}
$$


Since $\Gamma \backslash G$ is compact, Lemma 4.2(ii) implies that $Q:=\pi_{\lambda}^{-\omega}\left(\operatorname{Exp}\left(\frac{1}{2} X\right) G\right) \cdot v^{\Gamma}$ is compact in $\mathcal{H}_{\lambda}$. Thus we get

$$
\left|f_{v}\left(\Gamma s_{n}\right)\right| \leq \sup _{u \in Q}\left|\left\langle\pi_{\lambda}\left(h_{n}\right) \cdot w, \pi_{\lambda}\left(\operatorname{Exp}\left(\frac{1}{2} X+X_{n}\right)\right) \cdot u\right\rangle\right| .
$$

As $\Gamma s_{n}$ tends to infinity in $\Gamma \backslash \bar{S}$ we have $h_{n} \rightarrow \infty$ in $G$ or $X_{n} \rightarrow \infty$ in $\bar{C}$ (cf. Lemma 4.5(ii)). Case 1: $X_{n} \rightarrow \infty$. Then $\frac{1}{2} X+X_{n} \rightarrow \infty$ in $i \bar{C}$ and we have

$$
\left\|\pi_{\lambda}\left(\operatorname{Exp}\left(\frac{1}{2} X+X_{n}\right)\right)\right\|=\sup _{w \in \mathcal{W}_{\mathfrak{k}}} e^{w \cdot \lambda\left(\frac{1}{2} X+X_{n}\right)}
$$

(cf. [Kr99, Lemma 4.13]). Thus $\lambda \in i$ int $C^{\star}$ and the fact that $C$ is $\mathcal{W}_{\mathfrak{k}}$-invariant (and hence $i$ int $\left.C^{\star}\right)$ shows that $\left\|\pi_{\lambda}\left(\operatorname{Exp}\left(\frac{1}{2} X+X_{n}\right)\right)\right\| \rightarrow 0$. It follows from (4.2) that

$$
\lim _{n \rightarrow \infty}\left|f_{v}\left(\Gamma s_{n}\right)\right| \leq\|w\| \cdot \sup _{u \in Q}\|u\| \cdot \lim _{n \rightarrow \infty}\left\|\pi_{\lambda}\left(\operatorname{Exp}\left(\frac{1}{2} X+X_{n}\right)\right)\right\|=0
$$

Case 2: $h_{n} \rightarrow \infty$. We may assume that $X_{n}$ stays in a compact subset of $i \bar{C}$ because of Case 1 . Then $\pi_{\lambda}\left(\operatorname{Exp}\left(\frac{1}{2} X+X_{n}\right)\right) \cdot Q$ stays in a compact subset $Q^{\prime} \supseteq Q$ of $\mathcal{H}_{\lambda}$ and (4.2) implies that

$$
\left|f_{v}\left(\Gamma s_{n}\right)\right| \leq \sup _{u \in Q^{\prime}}\left|\left\langle\pi_{\lambda}\left(h_{n}\right) \cdot w, u\right\rangle\right| .
$$

Note that $\left(\pi_{\lambda}, \mathcal{H}_{\lambda}\right)$ has compact kernel, since $G$ is a (CA)Lie group, $Z(G)$ is compact and $\lambda \in i$ int $C^{\star}$ (cf. the proof of [Kr98a, Prop. V.7]). Hence the facts that $\left(\pi_{\lambda}, \mathcal{H}_{\lambda}\right)$ is irreducible and $G$ is a (CA)-Lie group imply that Mayer's generalization of the Howe-Moore Theorem (cf. [Ma97, Prop. 3.4], [HoMo79, Th. 5.1]) applies and so $\lim _{n \rightarrow \infty}\left|f\left(\Gamma s_{n}\right)\right|=0$.

\section{Separating holomorphic functions}

Now we are ready to prove the main result in this section. Recall from [Ko98, Ch. 3, §2] the definition of the Caratheodory semimetric and note that the Caratheodory semimetric is a metric if and only if the bounded holomorphic functions separate the points.

Theorem 4.7. (Separation of Points) Let $S$ be a complex Ol'shanskiu semigroup associated to a (CA)-Lie group $G$. Let $\Gamma<G$ be an arbitrary discrete subgroup of $G$. Then the following assertions hold:

(i) For every $z_{1}, z_{2} \in \Gamma \backslash S, z_{1} \neq z_{2}$, there exists an $f \in \mathcal{B}^{1}(\Gamma \backslash S)$ such that $0 \neq f\left(z_{1}\right) \neq f\left(z_{2}\right)$. In particular $\operatorname{Hol}(\Gamma \backslash S)$ separates the points of $\Gamma \backslash S$.

(ii) If $\Gamma<G$ is cocompact, then the bounded holomorphic functions on $\Gamma \backslash S$ separate the points. In particular, the Caratheodory semimetric on $\Gamma \backslash S$ is a metric.

Proof. (i) The proof uses a trick which was used by Siegel in order to show that the Siegel modular forms separate the points on $G / K$ (cf. [Fr83]).

Let $C_{0}(\Gamma \backslash \bar{S})$ denote the algebra of continuous function on $\Gamma \backslash \bar{S}$ which vanish at infinity. Then it follows from [Kr98a, Prop. II.4(i)(b)] and a slight adapation of [Kr98a, Lemma V.13] that $\mathcal{B}^{1}(S) \cap C_{0}(\bar{S})$ separates the points of $S$.

Let now $\Gamma s_{1}, \Gamma s_{2} \in \Gamma \backslash S$ be two different points. Let $f \in \mathcal{B}^{1}(S) \cap C_{0}(\bar{S})$ be such that

$$
m:=\sup _{\gamma \in \Gamma}\left\{\left|f\left(\gamma s_{1}\right)\right|,\left|f\left(\gamma s_{2}\right)\right|\right\}>0 .
$$


By Proposition 3.4 we know that for every $f \in \mathcal{B}^{1}(S)$ and $s \in S$ one has

$$
\left|f^{\Gamma}(s)\right| \leq \sum_{\gamma \in \Gamma}|f(\gamma s)|<\infty
$$

and $f^{\Gamma} \in \mathcal{B}^{1}(\Gamma \backslash S)$.

Hence the supremum $m$ is actually attained and without loss of generality we may assume that $m=1=f\left(s_{1}\right)$. Let now

$$
\Gamma_{0}:=\left\{\gamma \in \Gamma \backslash\{\mathbf{1}\}:\left|f\left(\gamma s_{1}\right)\right|=1 \quad \text { or } \quad\left|f\left(\gamma s_{2}\right)\right|=1\right\}
$$

and note that $\Gamma_{0}$ is a finite set by (4.3). Since $\mathcal{B}^{1}(S) \cap C_{0}(\bar{S})$ separates the points of $S$ we find a $g \in \mathcal{B}^{1}(S) \cap C_{0}(\bar{S})$ such that

$$
g\left(s_{1}\right)=1 \quad \text { and } \quad g\left(\gamma s_{1}\right)=g\left(\gamma s_{2}\right)=0 \quad\left(\forall \gamma \in \Gamma_{0}\right) .
$$

For every $n \in \mathbb{N}$ we now consider the function

$$
F_{n}(s)=g(s) f^{n}(s)
$$

and note that $F_{n} \in \mathcal{B}^{1}(S)$. Averaging over $\Gamma$ we obtain that

$$
\left(F_{n}\right)^{\Gamma}(\Gamma s)=\sum_{\gamma \in \Gamma} g(\gamma s) f^{n}(\gamma s)
$$

for all $s \in S$. Note that $\left(F_{n}\right)^{\Gamma} \in \mathcal{B}^{1}(\Gamma \backslash S)$. Now we have

$$
\left|\left(F_{n}\right)^{\Gamma}\right|\left(\Gamma s_{2}\right) \leq \sum_{\gamma \in \Gamma \backslash \Gamma_{0}}\left|g\left(\gamma s_{2}\right)\right|\left|f^{n}\left(\gamma s_{2}\right)\right|
$$

and we see that $\lim _{n \rightarrow \infty}\left(F_{n}\right)^{\Gamma}\left(\Gamma s_{2}\right)=0$ by (4.3) and $\left|f\left(\gamma s_{2}\right)\right|<1$ for all $\gamma \in \Gamma \backslash \Gamma_{0}$. On the other hand we have

$$
\left(F_{n}\right)^{\Gamma}\left(\Gamma s_{1}\right)=g\left(s_{1}\right) f\left(s_{1}\right)+\sum_{\gamma \in \Gamma \backslash \Gamma_{0}} g\left(\gamma s_{2}\right) f^{n}\left(\gamma s_{2}\right)=1+\sum_{\gamma \in \Gamma \backslash \Gamma_{0}} g\left(\gamma s_{2}\right) f^{n}\left(\gamma s_{2}\right)
$$

and by the same reason as before we have $\lim _{n \rightarrow \infty}\left(F_{n}\right)^{\Gamma}\left(\Gamma s_{1}\right)=1$. Hence there exists an $n \in \mathbb{N}$ such that $\left(F_{n}\right)^{\Gamma}\left(\Gamma s_{1}\right) \neq\left(F_{n}\right)^{\Gamma}\left(\Gamma s_{2}\right)$ proving (i)

(ii) In view of the proof of (i), this follows from Proposition 4.4.

Remark 4.8. By Neeb's version of the Gelfand-Raikov theorem (cf. [Ne99, Th. XI.5.1]) for complex Ol'shanskiú semigroups, the bounded holomorphic functions on $S$ separate the points. It follows that $S$ is hyperbolic complex. Hence $\Gamma \backslash S$ is hyperbolic complex for arbitrary discrete subgroups $\Gamma<G$ by [Sh92, §20, Th. 1]. To compare this with the results above notice that Theorem 4.7(ii) is a stronger statement than that $\Gamma \backslash S$ is hyperbolic complex. 
Example 4.9. (a) Let $G$ be a linear Hermitian Lie group. Then by a theorem of Borel (cf. [Bo63, Th. C], [Ra72, Th. 14.1]) there exist cocompact lattices $\Gamma<G$. Further the Lie algebra of $\mathfrak{g}$ admits pointed open convex $\operatorname{Ad}(G)$-invariant elliptic cones $\varnothing \neq W \subseteq \mathfrak{g}$. Set $S=\Gamma_{G}(W)$. Then by Theorem 4.8 the bounded holomorphic functions on $\Gamma \backslash S$ separate points.

(b) Let $\mathfrak{h}_{1}=\mathbb{R} X \oplus \mathbb{R} Y \oplus \mathbb{R} Z$ be the 3-dimensional Heisenberg algebra with relations $[X, Y]=Z$ and all other brackets vanishing. Then $\mathfrak{a}=\mathbb{R} T$ acts on $\mathfrak{h}$ by derivations via $[T, X]=Y$, $[T, Y]=-X$ and $[T, Z]=0$. Then $\mathfrak{g}=\mathfrak{h} \rtimes \mathfrak{a}$ is a four dimensional solvable Lie algebra, the so-called oscillator algebra. It is the basic example of a solvable Lie algebra admitting pointed open invariant convex cones $\varnothing \neq W \subseteq \mathfrak{g}$. Note that $\mathfrak{t}=\mathbb{R} Z \oplus \mathfrak{a}$ is a compactly embedded Cartan subalgebra of $\mathfrak{g}$.

Let $H_{1} \cong \mathbb{R}^{3}$ denote the simply connected Heisenberg group corresponding to $\mathfrak{h}_{1}$. We have a connected Lie group $G=H_{1} \rtimes \mathrm{S}^{1}$ with Lie algebra $\mathfrak{g}$. Then $\Gamma=\mathbb{Z}^{2} \times \frac{1}{2} \mathbb{Z} \subseteq H_{1}$ is a cocompact lattice in $G$ and hence the bounded holomorphic functions on $\Gamma \backslash S$ separate points by Theorem 4.8 .

(c) Now we give an example of a Lie group $G$ of mixed type, i.e., $G$ is neither reductive nor solvable. We define a quadratic form

$$
F: \mathbb{R}^{3} \rightarrow \mathbb{R}, \quad(x, y, z) \mapsto x^{2}+y^{2}-3 z^{2}
$$

and let $L:=\mathrm{SO}_{0}(F, \mathbb{R})$ denote the connected component of the special invariance group of $F$. Then $F$ does not represent zero in a non-trivial rational way so that $\Gamma_{L}:=\mathrm{SO}_{0}(F, \mathbb{Z})$ forms a cocompact lattice in $L$ (cf. [Bo69, 8.6, Ex. 1]).

Let $\langle\cdot, \cdot\rangle_{2,1}$ denote the non degenerate symmetric bilinear form on $\mathbb{R}^{3}$ induced by $F$. Then the prescription

$$
\Omega_{F}:\left(\mathbb{R}^{3} \times \mathbb{R}^{3}\right) \times\left(\mathbb{R}^{3} \times \mathbb{R}^{3}\right) \rightarrow \mathbb{R}, \quad\left((u, v),\left(u^{\prime}, v^{\prime}\right)\right) \mapsto\left\langle u, v^{\prime}\right\rangle_{2,1}-\left\langle u^{\prime}, v\right\rangle_{2,1}
$$

defines an $L$-invariant symplectic form on $\mathbb{R}^{3} \times \mathbb{R}^{3}$ (we let $L$ act diagonally). Associated to $\Omega_{F}$ we build the nilpotent Lie algebra $\mathfrak{u}:=\left(\mathbb{R}^{3} \times \mathbb{R}^{3}\right) \times \mathbb{R}$ with bracket

$$
\left[((u, v), z),\left(\left(u^{\prime}, v^{\prime}\right), z^{\prime}\right)\right]=\left(0, \Omega_{F}\left((u, v),\left(u^{\prime}, v^{\prime}\right)\right)\right) .
$$

Note that $\mathfrak{u}$ is isomorphic to the 7-dimensional Heisenberg algebra $\mathfrak{h}_{3}$. Let $U$ denote a simply connected Lie group with Lie algebra $u$. Then $U \cong\left(\mathbb{R}^{3} \times \mathbb{R}^{3}\right) \times \mathbb{R}$ and $\Gamma_{U}:=\left(\mathbb{Z}^{3} \times \mathbb{Z}^{3}\right) \times \frac{1}{2} \mathbb{Z}$ is a cocompact lattice in $U$ which is stable under $\Gamma_{L}$. Thus $\Gamma:=\Gamma_{U} \rtimes \Gamma_{L}$ is a cocompact lattice in the semidirect product $G:=U \rtimes L$.

Note that there is an embedding of $\mathfrak{g}$ into the Jacobi algebra $\mathfrak{h}_{3} \rtimes \mathfrak{s p}(3, \mathbb{R})$ sending $\mathfrak{u}$ isomorphically onto $\mathfrak{h}_{3}$ and $\mathfrak{l}:=\mathbf{L}(L)$ into $\mathfrak{s p}(3, \mathbb{R})$. In view of [Ne99a, Ch. VII], this embedding guarantees us the existence of non-trivial pointed $\operatorname{Ad}(G)$-invariant open convex cones $W \subseteq \mathfrak{g}$. Now the bounded holomorphic functions on $\Gamma \backslash \Gamma_{G}(W)$ separate points by Theorem 4.8.

\section{The Stein property of $\Gamma \backslash S$ for $\Gamma \backslash G=\operatorname{Sl}(2, \mathbb{Z}) \backslash \mathrm{Sl}(2, \mathbb{R})$}

We let the group $G=\operatorname{Sl}(2, \mathbb{R})$ act on the upper halfplane $\Pi^{+}:=\{z \in \mathbb{C}: \operatorname{Im} z>0\}$ by means of Möbius transformations:

$$
g . z=\frac{a z+b}{c z+d} \quad g=\left(\begin{array}{cc}
a & b \\
c & d
\end{array}\right) \in G, z \in \Pi^{+} .
$$

Note that $G_{\mathbb{C}}=\operatorname{Sl}(2, \mathbb{C})$. The compression semigroup of $\Pi^{+}$

$$
\bar{S}:=\left\{g \in G_{\mathbb{C}}: g \cdot \Pi^{+} \subseteq \Pi^{+}\right\}
$$


defines a closed complex Ol'shanskiur semigroup with interior

$$
S:=\left\{g \in G_{\mathbb{C}}: g \cdot \overline{\Pi^{+}} \subseteq \Pi^{+}\right\}
$$

Let $U=\left(\begin{array}{cc}0 & 1 \\ -1 & 0\end{array}\right)$ be the infinitesimal generator of the maximal compact subgroup $K=$ $\operatorname{SO}(2, \mathbb{R})$ of $G$. Set $\left.\mathbb{R}^{-}=\right]-\infty, 0[$. Then it is easily shown that

$$
S=G \exp \left(i \mathbb{R}^{-} U\right) G=G \exp (i W)
$$

with $W=\operatorname{Ad}(G) \cdot \mathbb{R}^{-} U$ the lower light cone in $\mathfrak{g}=\mathfrak{s l}(2, \mathbb{R})$. Note that $W$ and $-W$ are the only non-trivial $\operatorname{Ad}(G)$-invariant pointed convex cones in $\mathfrak{g}$.

Let $\Gamma=\operatorname{Sl}(2, \mathbb{Z})$ be the modular group. For $z \in \Pi^{+}$set $q:=e^{2 \pi i z}$. Then the discriminant

$$
\Delta: \Pi^{+} \rightarrow \mathbb{C}, \quad z \mapsto q \prod_{n=1}^{\infty}\left(1-q^{n}\right)^{24}
$$

defines a modular form of weight 12 , i.e., $\Delta$ is holomorphic, vanishes at infinity and satisfies

$$
\left(c_{\gamma} z+d_{\gamma}\right)^{-12} \Delta(\gamma \cdot z)=\Delta(z) \quad z \in \Pi^{+}, \gamma=\left(\begin{array}{ll}
a_{\gamma} & b_{\gamma} \\
c_{\gamma} & d_{\gamma}
\end{array}\right)
$$

For $g=\left(\begin{array}{ll}a & b \\ c & d\end{array}\right) \in G$ and $z \in \Pi^{+}$we define

$$
\mu(g, z):=c z+d
$$

and note that $\mu$ is a cocycle, i.e., $\mu\left(g_{1} g_{2}, z\right)=\mu\left(g_{1}, g_{2} . z\right) \mu\left(g_{2}, z\right)$ for all $g_{1}, g_{2}, \in G, z \in \Pi^{+}$. Then the description

$$
f_{\Delta}: G \rightarrow \mathbb{C}, \quad g \mapsto \mu(g, i)^{12} \Delta(g . i)
$$

defines a left $\Gamma$-invariant analytic function on $G$ (cf. [Bo66]). Since $\bar{S}$ compresses $\Pi^{+}$, we see that $\mu$ extends to a continuous function $\mu(s, z), s \in \bar{S}, z \in \Pi^{+}$, with no zeros. By the same reason the prescription $s \mapsto \Delta(s . i)$ defines a continuous function on $\bar{S}$. Hence we can analytically continue $f_{\Delta}$ to a continuous function

$$
F_{\Delta}: \bar{S} \rightarrow \mathbb{C}, \quad s \mapsto \mu(s, i)^{12} \Delta(s . i)
$$

which is holomorphic when restricted to $S$. Note that $F_{\Delta}$ is left $\Gamma$-invariant and hence factors to a function on $\Gamma \backslash \bar{S}$, also denoted by $F_{\Delta}$.

It is a consequence of $[\mathrm{KO} 01, \mathrm{Th} .3 .2]$ that $F_{\Delta}$ vanishes at infinity on $\Gamma \backslash S$, i.e.,

$$
\lim _{\substack{\Gamma s \rightarrow \infty \\ \Gamma s \in \Gamma \backslash \bar{S}}} F_{\Delta}(\Gamma s)=0
$$

Let us briefly scetch the proof of this fact. The discriminant $\Delta$ is bounded on a fundamental domain for $\Gamma$ in $\Pi^{+}$. Hence it is sufficient to show that $\lim _{\substack{s \rightarrow \infty \\ s \in \bar{S}}} \mu(s, i)=0$. For $G=\operatorname{Sl}(2, \mathbb{R})$ this can be seen by direct calculation; more generally it follows from the fact that the middle projection $\kappa: P^{-} K_{\mathbb{C}} P^{+} \rightarrow K_{\mathbb{C}}$ restricted to $\bar{S}$ is a proper map and has image $\kappa(\bar{S})=K \exp \left(i \mathbb{R}^{-} U\right)$ (cf. $\left[\right.$ KO01, Prop. 1.2, Cor. 2.4]). We note that $F_{\Delta}$ has no zeroes on $\Gamma \backslash \bar{S}$. Hence $\frac{1}{\left|F_{\Delta}\right|}$ defines a continuous plurisubharmonic function on $\Gamma \backslash \bar{S}$ and (5.1) implies that

$$
\lim _{\substack{\Gamma s \rightarrow \infty(i n \Gamma \backslash \bar{S}) \\ \Gamma s \in \Gamma \backslash S}} \frac{1}{\left|F_{\Delta}\right|}(\Gamma s)=\infty .
$$


Theorem 5.1. Let $S$ be a complex Ol'shanskì semigroup associated to $G=\operatorname{Sl}(2, \mathbb{R})$ and let $\Gamma=\operatorname{Sl}(2, \mathbb{Z})$. Then $\Gamma \backslash S$ is Stein.

Proof. In view of Grauert's solution of the Levi problem (cf. Theorem 2.2), it is sufficient to prove the existence of a strictly plurisubharmonic exhaustion function of $\Gamma \backslash S$.

Let $\psi$ be the non-negative biinvariant plurisubharmonic function on $S$ from Theorem 2.4. Then $\psi$ factorizes to a function on $\Gamma \backslash S$ which we also denote by $\psi$. Then

$$
\varphi:=\psi+\frac{1}{\left|F_{\Delta}\right|}
$$

defines a strictly plurisubharmonic function on $\Gamma \backslash S$. To conclude the proof of the theorem it suffices to show that $\varphi$ is proper. Let $\left(z_{n}\right)_{n \in \mathbb{N}}$ be a sequence in $\Gamma \backslash S$ leaving every compact subset of $\Gamma \backslash S$. Then we either have $z_{n} \rightarrow z \in(\Gamma \backslash \bar{S})-(\Gamma \backslash S)$ or $z_{n} \rightarrow \infty$ in $\Gamma \backslash \bar{S}$. In the first case we have $\psi\left(z_{n}\right) \rightarrow \infty$ by Theorem 2.4 while in the latter case $\lim _{n \rightarrow \infty} \frac{1}{\left|F_{\Delta}\left(z_{n}\right)\right|}=\infty$ by $(5.2)$. This concludes the proof of the theorem.

Remark 5.2. (a) Note that by Theorem 2.3 now also for all subgroups $\Gamma_{0}<\operatorname{Sl}(2, \mathbb{Z})$ the manifold $\Gamma_{0} \backslash S$ is Stein.

(b) Let $G$ be a hermitian Lie group and $\Gamma<G$ a lattice. We claim that there exists no holomorphic cusp forms on $G / K$ without zeros if $\Gamma<G$ is cocompact or if the real rank $\operatorname{rank}(G)$ of $G$ is strictly larger than one. Since if $f$ were a holomorphic cusp form without zeros, then $\frac{1}{f}$ would again be an automorphic form. If $\Gamma<G$ is cocompact, this is clear, and if $\Gamma<G$ is not cocompact and $\operatorname{rank}(G)>1$, then this is implied by the Koecher-principle (cf. [Fr83, Ch. I, Hilfssatz 3.5]). Now $\frac{1}{f}$ would be of "negative weight" which is impossible since there are no automorphic forms of negative weight (if $\Gamma<G$ is cocompact, this is a consequence of the Plancherel Theorem for $L^{2}(\Gamma \backslash G)$ and in the non-cocompact case this can be proved as in [Fr83, Ch. I, Satz 3.13]).

Therefore our construction for $\mathrm{Sl}(2, \mathbb{R})$ cannot be generalized to arbitrary hermitian groups nor to the case of cocompact subgroups.

Conjecture 5.3. We conjecture that $\Gamma \backslash S$ is Stein for all complex Ol'shanskiu semigroups and all discrete subgroups $\Gamma<G$. In view of the proof of Theorem 5.1, it would be sufficient to find a holomorphic function on $\Gamma \backslash S$ with no zeros and which vanishes at infinity on $\Gamma \backslash \bar{S}$. If $\Gamma<G$ is cocompact, then we have seen in Theorem 4.6 that all matrix coefficients $f_{v}(\Gamma s)=\left\langle\pi(s) \cdot v, v^{\Gamma}\right\rangle$ for $v \in \mathcal{H}^{\omega}$ vanish at infinity. The difficulty is to show that there exists a certain $v \in \mathcal{H}^{\omega}$ so that $f_{v}$ has no zeros. As we pointed out in Remark 5.2, this can never happen if $v$ is a highest weight vector. But it is likely to be true that $f_{v}$ has no zeros for all $v \in \pi_{\lambda}(S) \cdot v^{\Gamma}$, as the reproducing kernel

$$
K(\Gamma s, \Gamma t)=\left\langle\pi\left(t^{*}\right) \cdot v^{\Gamma}, \pi\left(s^{*}\right) \cdot v^{\Gamma}\right\rangle
$$

of the realiztion of $(\pi, \mathcal{H})$ in $\operatorname{Hol}(\Gamma \backslash S)$ should be zero-free by general principles. However, this seems to be a very challenging problem, even for $G=\operatorname{Sl}(2, \mathbb{R})$.

\section{References}

[Ach99] Achab, D., Espace de Hardy pour les quotients $\Gamma \backslash G$, Math. Z. 230(1) (1999), $21-45$.

[BaOt73] Barth, W., and M. Otte, Invariante holomorphe Funktionen auf reduktiven LieGruppen, Math. Ann. 201 (1973), 97-112. 
Discrete group actions on Stein domains in complex Lie groups

[Bo63] Borel, A., Compact Clifford-Klein forms of symmetric spaces, Topology 2 (1963), 111-122.

[Bo66] -, Introduction to automorphic forms, Proc. Sympos. Pure Math. 9 (1966), Amer. Math. Soc., Providence, RI, 199-210.

[Bo69] —, "Introduction aux groupes arithmétiques", Hermann, Paris, 1969.

[Fr83] Freitag, E., "Siegelsche Modulfunktionen", Grundlehren der Mathematischen Wissenschaften 254, Springer, 1983.

[GiHu78] Gilligan, B., and A. Huckleberry, On non-compact complex nil-manifolds, Math. Ann. 238 (1978), 39-49.

[Hel78] Helgason, S., "Lie Groups, Differential Geometry and Symmetric Spaces", Academic Press, London, 1978.

[HiKr98] Hilgert, J., and B. Krötz, The Plancherel Theorem for invariant Hilbert spaces, Math. Z. 37(1) (2001), 31-59.

[HiKr99] Hilgert, J., and B. Krötz, Representation, characters, and spherical functions associated to causal symmetric spaces, J. Funct. Anal. 169 (1999), 357-390.

[HiKr01] _, The Plancherel Theorem for invariant Hilbert spaces, Math. Z. 37(1) (2001), $31-59$.

[HiNe93] Hilgert, J., and K.-H. Neeb, "Lie semigroups and their Applications", Lecture Notes in Math. 1552, Springer, 1993.

[Hö73] Hörmander, L., "An introduction to complex analysis in several variables", North-Holland, 1973.

[HoMo79] Howe, R., and C. Moore, Asymptotic Properties of Unitary Representations, J. Funct. Anal. 32 (1979), 72-96.

[Ko98] Kobayashi, S., "Hyperbolic complex spaces", Grundlehren der mathematischen Wissenschaften 318, Springer 1998.

[Kr98a] Krötz, B., On Hardy and Bergman spaces on complex Ol'shanskŭ semigroups, Math. Ann. 312 (1998), 13-52.

[Kr98b] - On the dual of complex Ol'shanskiu semigroups, Math. Z. 237 (2001), 505529.

[Kr99] - The Plancherel Theorem for Biinvariant Hilbert Spaces, Publ. RIMS 35 (1) (1999), 91-122.

[Kr01] - Formal dimension for semisimple symmetric spaces, Comp. Math. 125 (2001), 155-191.

[KNÓ97] Krötz, B., K.-H. Neeb, and G. Ólafsson, Spherical Representations and Mixed Symmetric Spaces, Represent. Theory 1 (1997), 424-461.

[KNÓ01] -, Spherical Functions on Mixed Symmetric Spaces, Represent. Theory 5 (2001), 43-92.

[KO01] Krötz, B., and M. Otto, Vanishing properties of analytically continued matrix coefficients, J. Lie Theory, to appear.

[Lo84] Loeb, J.-J., Fonctions plurisousharmoniques sur un groupe de Lie complexe invariantes par une forme rèelle, C. R. Acad. Sci. Paris Sèr. I Math. 299 (1984), no. 14, 663-666.

[MaMo60] Matsushima, Y., and A. Morimoto, Sur certain espaces fibrés holomorphe sur une variété de Stein, Bull. Soc. Math. France 88(1960), 137-155.

[Ma97] Mayer, M., Asymptotics of Matrix Coefficients and Closures of Fourier-Stieltjes Algebras, J. Funct. Anal. 143(1), (1997), 42-54. 
[Ne97] Neeb, K.-H., A general non-linear convexity theorem, Forum Math. 9 (1997), 613-640.

[Ne98] - On the complex and convex geometry of Ol'shanskir semigroups, Ann. Inst. Fourier 48(1) (1998), 149-203.

[Ne99a] -, "Holomorphy and Convexity in Lie Theory," Expositions in Mathematics 28, de Gruyter, 1999.

[Ne99b] - On the complex geometry of invariant domains in complexified symmetric spaces, Ann. Inst. Fourier 49(1) (1999), 177-225.

[Ols82] Ol'shanskiŭ, G. I., Invariant cones in Lie algebras, Lie semigroups, and the holomorphic discrete series, Funct. Anal. and Appl. 15, 275-285 (1982).

[Ra72] Raghunathan, M. S., "Discrete Subgroups of Lie groups", Ergebnisse der Mathematik 68, Springer, 1972.

[Sh92] Shabat, B. V., "Introduction to Complex Analysis, Part II: Functions of Several Variables", Amer. Math. Soc., Providence, Rhode Island, 1992.

[Sta86] Stanton, R. J., Analytic Extension of the holomorphic discrete series, Amer. J. Math. 108 (1986), 1411-1424.

[WaWo93] Wallach, N., and J. A. Wolf, Completeness of Poincaré series for automorphic forms associated to the integrable discrete series, Representation theory of reductive groups (Park City, Utah, 1982), 265-281, Progr. Math., 40, Birkhuser Boston, Boston, Mass., 1983.

[WeWo77] Wells, R. O., and J. A. Wolf, Poincaré series and automorphic cohomology on flag domains, Ann. of Math. (2) 105 (1977), no. 3, 397-448.

Dehbia Achab

Université Pierre et Marie Curie

Institut de Mathématiques

Analyse Algébrique - Case 82

Tour 46-0

4 , place Jussieu

F-75252 Paris Cedex 05

France
Frank Betten

Mathematisches Institut

Universität Göttingen

Bunsenstraße 3-5

D-37073 Göttingen

Germany

Bernhard Krötz

The Ohio State University

Department of Mathematics

231 West 18th Avenue

Columbus, OH 43210-1174

USA 